\title{
Exosomes Derived from Bone Mesenchymal Stem Cells Repair Traumatic Spinal Cord Injury by Suppressing the Activation of A1 Neurotoxic Reactive Astrocytes
}

\author{
Wei Liu, ${ }^{1, *}$ Yongxiang Wang, ${ }^{2, *}$ Fangyi Gong,, ${ }^{1, *}$ Yuluo Rong, ${ }^{1}$ Yongjun Luo, Pengyu Tang, Zheng Zhou, \\ Zhimin Zhou, Tao Xu, Tao Jiang, Siting Yang, Guoyong Yin, Jian Chen, Jin Fan, and Weihua Cai ${ }^{1}$
}

\begin{abstract}
Mesenchymal stem cell (MSC) transplantation is now considered as an effective treatment strategy for traumatic spinal cord injury (SCI). However, several key issues remain unresolved, including low survival rates, cell dedifferentiation, and tumor formation. Recent studies have demonstrated that the therapeutic effect of transplanted stem cells is primarily paracrine mediated. Exosomes are an important paracrine factor that can be used as a direct therapeutic agent. However, there are few reports on the application of exosomes derived from bone MSCs (BMSCs-Exos) in treating SCI. In this study, we demonstrated that BMSCs-Exos possessed robust proangiogenic properties, attenuated neuronal cells apoptosis, suppressed glial scar formation, attenuated lesion size, suppressed inflammation, promoted axonal regeneration, and eventually improved functional behavioral recovery effects after traumatic SCI. Briefly, lesion size was decreased by nearly $60 \%$, neuronal apoptosis was attenuated by nearly $70 \%$, glial scar formation was reduced by nearly $75 \%$, average blood vessel density was increased by nearly $60 \%$, and axonal regeneration was increased by almost $80 \%$ at day 28 after SCI in the BMSC-Exos group compared to the control group. Using a series of in vitro functional assays, we also confirmed that treatment with BSMCsExos significantly enhanced human umbilical vein endothelial cell proliferation, migration, and angiogenic tubule formation, attenuated neuronal cells apoptosis, and suppressed nitric oxide release in microglia. Moreover, our study demonstrated that administration of BMSCs-Exos suppressed inflammation efficiently after traumatic SCI and suppressed activation of A1 neurotoxic reactive astrocytes. In conclusion, our study suggested that the application of BMSCs-Exos may be a promising strategy for traumatic SCI.
\end{abstract}

Keywords: bone mesenchymal stem cells; exosomes; neurotoxic reactive astrocytes; spinal cord injury

\section{Introduction}

$\mathbf{T}$ RAUMATIC SPINAL CORD INJURY (SCI) is a major medical problem, which affects approximately 180,000 patients worldwide each year, ${ }^{1}$ often resulting in motor deficits, bladder dysfunctions, and even death. ${ }^{2}$ SCI is the result of an initial contusion or compression injury followed by a secondary injury that leads to activation of neuroinflammation, microvascular disruption, formation of glial scars, and upregulation of inhibitory factors, such as chondroitin sulfate proteoglycans (CSPGs) and myelinassociated inhibitors (MAIs), which limit axonal extension and further behavioral recovery. ${ }^{3,4}$ Current studies on SCI mainly focused on rebuilding functional synapses by promoting the intrinsic growth capacity of neurons, downregulating inhibitory factors, reducing neuroinflammation, removing extrinsic barriers, and minimizing lost spinal tissues. ${ }^{5-7}$ Besides, biomaterials are getting more and more attention in SCI therapy for their unique advantage in promoting cell survival and differentiation without the need of catheters or repeated injections. ${ }^{8,9}$ However, many of these studies only showed limited and unsatisfactory therapeutic efficacy. ${ }^{10}$

Cell transplantation might be a promising strategy to improve recovery of motor, sensory, and/or autonomic functions after SCI. ${ }^{6,11,12}$ Because of their potential and promising neuroprotective properties, bone mesenchymal stem cells (BMSCs) have attracted particular interest. ${ }^{11,13-15}$ Several studies suggested that BMSCs provide a microenvironment conducive to cellular survival through secreting neurotrophic factors, neuroprotective cytokines, and antiinflammatory molecules, as well as reducing production of stressassociated proteins, reactive oxygen, and proinflammatory cytokines. ${ }^{14,15}$ However, direct transplantation of stem cells to target

\footnotetext{
${ }^{1}$ Department of Orthopaedics, the First Affiliated Hospital of Nanjing Medical University, Nanjing, China.

${ }^{2}$ Department of Orthopaedics, Clinical Medical College of Yangzhou University, Subei People's Hospital of Jiangsu Province, Yangzhou, China.

${ }^{3}$ Department of Orthopaedics, Wuxi People's Hospital Affiliated to Nanjing Medical University, Wuxi, Jiangsu, China.

${ }^{4}$ Department of Anesthesia, the First Affiliated Hospital of Nanjing Medical University, Nanjing, China.

*These authors contributed equally to this work.
} 


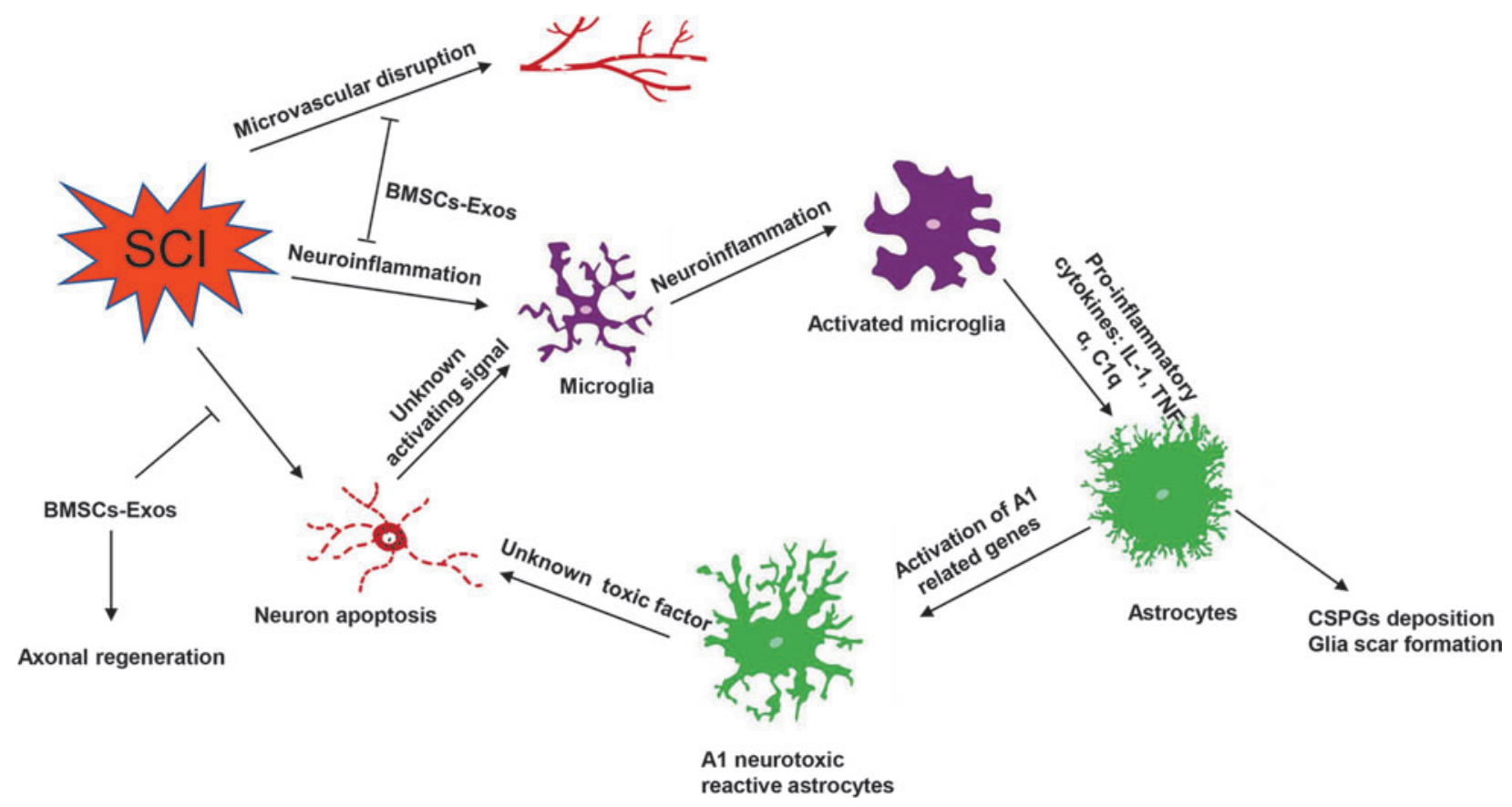

FIG. 1. Diagram illustrating the proposed mechanism of administration of BMSC-Exos after traumatic SCI. BMSCs-Exos possessed robust proangiogenic properties, attenuated neuronal cell apoptosis, suppressed glial scar formation, suppressed inflammation, promoted axonal regeneration, and eventually improved functional behavioral recovery after traumatic SCI. Especially, administration of BMSCExos suppressed activation of A1 neurotoxic reactive astrocytes. BMSCs-Exos, bone mesenchymal stem cells/exosomes; CSPGs, chondroitin sulfate proteoglycans; IL, interleukin; SCI, spinal cord injury; TNF, tumor necrosis factor. Color image is available online at www.liebertpub.com/neu

tissues remains challenging. For example, it has been demonstrated that transplanted stem cells exhibit a low survival rate in ischemic tissue. ${ }^{16}$ Other risks, such as cell dedifferentiation, immune rejection, and tumor formation, further limit the clinical application of direct transplantation of stem cells for SCI. ${ }^{17,18}$

Recent studies have demonstrated that transplanted stem cells exert therapeutic effects mainly by a paracrine mechanism, and that exosomes play an important and major role in this process. ${ }^{19,20}$ Exosomes, an important component of cell paracrine secretion, are nano-sized liposomes that are originated from invagination of the endosomal membrane in multi-vesicular bodies with a diameter of $20-150 \mathrm{~nm} .^{21,22}$ They are then released into extracellular space through fusion with the plasma membrane, which protects their contents from degradation or destruction. Exosomes are involved in transportation of functional biochemicals, such as cytokines, RNA, and proteins, and thus play a crucial role in cell communication. Specific surface ligands of an exosome enable it to bind to a target cell, deliver or transfer biomessages and relevant genetic proteins into the target cell, and eventually regulate a particular biological function. ${ }^{23,24}$ It has been confirmed that transplantation of exosomes shows similar therapeutic effects and functional properties as direct stem cell transplantation with no apparent adverse effects. ${ }^{25-27}$ Recently, a study demonstrated that neurovascular remodeling and significant functional behavioral recovery was achieved with the application of exosomes derived from mesenchymal stem cells (MSCs) in a rat stroke model. ${ }^{28}$ Similarly, a recent study showed that cognitive impairments caused by traumatic brain injury were remarkably reduced by systemic administration of extracellular vesicles originated from MSCs. ${ }^{29}$ Thus, we suggest that exosomes derived from BMSCs may also promote functional behavioral recovery after traumatic SCI.
We therefore studied the application of BMSCs-Exos as a regenerative medicine in traumatic SCI both in vitro and in vivo and, for the first time, outlined a potential mechanism that may suppress neuroinflammation, regulate axonal regeneration, and, as a result, promote functional recovery in rats after SCI (Fig. 1).

\section{Methods \\ Isolation and purification of bone mesenchymal stem cells/exosomes}

Exosomes were isolated from BMSCs supernatant as previously described. ${ }^{30,31}$ Culture medium was replaced with an exosomedepleted fetal bovine serum (FBS)-containing medium (EXO-FBS250A-1; System Biosciences, Mountain View, CA) for an additional $48 \mathrm{~h}$ after BMSCs reached $\sim 80 \%$ confluency. Medium was then collected and centrifuged at $300 \mathrm{~g}$ for $10 \mathrm{~min}$, followed by centrifugation at $2000 \mathrm{~g}$ for $10 \mathrm{~min}$ at $4^{\circ} \mathrm{C}$. After centrifugation, the cell supernatant was filtered using a $0.22-\mu \mathrm{m}$ filter sterilize Steritop $^{\mathrm{TM}}$ (Millipore, Burlington, MA) to remove whole cells and cellular debris. Afterward, the supernatant was transferred to the upper compartment of an Amicon Ultra-15 Centrifugal Filter Unit (Millipore) and centrifuged at $4000 \mathrm{~g}$ at $4^{\circ} \mathrm{C}$ until the volume in the upper compartment was reduced to nearly $200 \mu \mathrm{L}$. The ultrafiltration liquid was then washed twice with phosphate-buffered saline (PBS) and again re-ultrafiltrated to $200 \mu \mathrm{L}$. For exosome purification, the liquid was loaded on top of a $30 \%$ sucrose $/ \mathrm{D}_{2} \mathrm{O}$ cushion in a sterile Ultra-Clear ${ }^{\mathrm{TM}}$ tube (Beckman Coulter, Brea, CA) and centrifuged at $100,000 \mathrm{~g}$ for $60 \mathrm{~min}$ at $4^{\circ} \mathrm{C}$ (Sorvall, Avanti J-26XP, fixed angle rotor; Beckman Coulter). The fraction containing BMSCs-Exos was recovered using an 18-G needle, diluted in PBS, and centrifuged at $4000 \mathrm{~g}$ at $4^{\circ} \mathrm{C}$ in centrifugal filter units until the final volume reached $200 \mu \mathrm{L}$. Exosomes were stored at $-80^{\circ} \mathrm{C}$ or used for another series of experiments. BMSCs-Exos protein 
content was determined by using a bicinchoninic acid assay (BCA; Thermo Fisher Scientific, Waltham, MA). Absorbance was read at $562 \mathrm{~nm}$ by using a microplate reader (ELx800; Bio-Tek Instruments, Inc., Winooski, VT).

\section{Identification of bone mesenchymal stem cells/ exosomes}

Nanosizer ${ }^{\mathrm{TM}}$ technology (Malvern Instruments, Malvern, UK) was applied to analyze the diameter distribution of BMSC-Exos. Morphology of the acquired exosomes was observed by transmission electron microscopy (TEM; Tecnai 12; Philips, Best, The Netherlands). Western blotting was used to examine the specific exosome surface markers, which was encapsulated into exosomes including CD9, CD63, and CD81.

\section{Cells and cell culture}

According to an established protocol, ${ }^{32}$ primary neurons were collected from embryonic (E16-E18) Sprague-Dawley (SD) rats. Briefly, cerebral cortices were isolated and dissociated with trypsin $(0.25 \%, \mathrm{w} / \mathrm{v}$; Thermo Fisher Scientific) for $20 \mathrm{~min}$. Neurons were seeded at a density of $5 \times 10^{4} / \mathrm{mL}$ for immunofluorescent staining in 24 -well culture plates and $1 \times 10^{6} / \mathrm{mL}$ for western blot assays in sixwell culture plates. Neurons were maintained in fresh neurobasal medium (Thermo Fisher Scientific) containing 2\% B27 (2\%, w/v; Thermo Fisher Scientific), $1 \%$ glutamine (Thermo Fisher Scientific), $100 \mathrm{IU} / \mathrm{mL}$ of penicillin, and $100 \mathrm{mg} / \mathrm{mL}$ of streptomycin. Half of the medium was changed every third day. After 5 days of cell culture, the obtained neurons were examined by microtubuleassociated protein-2 (MAP-2; 1:500, mouse immunoglobulin G [IgG]; Abcam, Cambridge, MA) under a fluorescence microscope (Supplementary Fig. S1A). (see online supplementary material at http://www.liebertpub.com)

According to an established protocol, ${ }^{33}$ bone marrow from adult male SD rats was first mechanically dissociated and cells were then washed and suspended in culture medium. After 3 days, cells that tightly adhered to culture flasks were considered as P0 MSCs. Afterward, BMSCs were cultured in a modified Eagle's medium (Hyclone, Logan, UT) containing 10\% FBS (Gibco Laboratory, Grand Island, NY) and penicillin/streptomycin on $25-\mathrm{cm}^{2}$ tissue culture flasks (Corning, St Louis, MO).

Primary microglia were harvested from post-natal day 3 rat pups as described previously. ${ }^{34}$ The obtained cells were examined by CD68 (1:300, mouse IgG1; EMD Millipore Corp., Burlington, MA) and ionized calcium-binding adapter molecule 1 (Iba1; 1:200, rabbit IgG; Abcam, UK) under a fluorescence microscope (Supplementary Fig. S1B). (see online supplementary material at http:// www.liebertpub.com)

The human umbilical vein endothelial cell (HUVEC) line was purchased from American Type Culture Collection (ATCC; Manassas, VA). Cell lines were cultured in Dulbecco's modified Eagle's medium/high glucose (Hyclone, UT, USA) containing 10\% FBS, and $1 \%$ penicillin-streptomycin.

\section{Cell viability assay}

Viability of HUVECs was evaluated with a Cell Counting Kit- 8 (CCK-8) assay (Dojindo, Kumamoto, Japan) to examine the effect of BMSCs-Exos on proliferation of HUVEC cells. After 0, 24, 48, 72 , and $96 \mathrm{~h}$ of incubation, wells were rinsed three times with $1 \times$ PBS. Afterward, CCK- 8 solution $(10 \mu \mathrm{L} ; 1: 10$ diluted $)$ in fresh culture medium was added and incubated for $2 \mathrm{~h}$ at $37^{\circ} \mathrm{C}$. Finally, optical absorbance was measured at $450 \mathrm{~nm}$ using an absorbance microplate reader (ELx800; Bio-Tek).

\section{Tube formation assay}

To assess the possible angiogenic property of BMSCs-Exos, HUVECs were seeded at $2.0 \times 10^{4}$ cells/well in a Matrigel-coated 96-well plate (Matrigel; BD Biosciences, San Jose, CA). Briefly, 96-well plates were covered with $50 \mu \mathrm{L}$ of Matrigel by using a precooled tip at $4^{\circ} \mathrm{C}$. Matrigel was then coated with $100 \mu \mathrm{L}$ of a suspension of HUVEC cells treated with or without $100 \mu \mathrm{g} / \mathrm{mL}$ of BMSCs-Exos after gelling for additional $30 \mathrm{~min}$ at $37^{\circ} \mathrm{C}$. Tube formation capacity was assessed by observing the polygonal structures formed $6 \mathrm{~h}$ after plating cells onto the Matrigel using a light microscope. Tube formations, including total tube length and total branch numbers, were carefully measured.

\section{Migration assays}

Transwell assay was used to analyze the effect of BMSCs-Exos on HUVECs migration. Briefly, $2 \times 10^{4}$ HUVEC cells were seeded into the upper chamber of a 24 -well transwell plate (pore size, $8 \mu \mathrm{m}$; Corning Incorporated, Corning, NY) after which $600 \mu \mathrm{L} /$ well of 0 or $100 \mu \mathrm{g} / \mathrm{mL}$ of BMSC-Exos were added to the lower chamber. After co-incubation for $24 \mathrm{~h}$, cells from the upper surface of filter membranes were wiped away with a cotton swab. Cells that migrated to the lower surface of the filter membrane were stained with $0.5 \%$ crystal violet for $1 \mathrm{~min}$. Migratory activity was assessed by observing the stained HUVEC cells under an optical microscope.

The scratch wound assay was performed to assess migration ability as previously described. ${ }^{35}$ Briefly, HUVECs were seeded into six-well plates at a density of $2 \times 10^{5}$ cells/well and then grown to $100 \%$ confluence. Afterward, the confluent layer of cells was scratched using a sterile $200-\mu \mathrm{L}$ pipette tip. After carefully washing cells with PBS three times, 0 or $100 \mu \mathrm{g} / \mathrm{mL}$ of BMSCs-Exos were added. Images were recorded at 0,12 , and $24 \mathrm{~h}$ after scratching of the confluent layer of cells.

\section{Nitric oxide assay}

Microglia $\left(2 \times 10^{5}\right.$ cells $\left./ \mathrm{mL}\right)$ were plated onto 96 -well plates and pre-treated with or without BMSCs-Exos $(100 \mu \mathrm{g} / \mathrm{mL}) 1 \mathrm{~h}$ before stimulation with $5 \mathrm{ng} / \mathrm{mL}$ of lipopolysaccharide (LPS; SigmaAldrich, St. Louis, MO). Supernatants were then collected and assessed for nitric oxide (NO) production by using Griess reagent (Promega, Madison, WI) every $24 \mathrm{~h}$ for 4 days. Briefly, supernatants were mixed with an equal volume of Griess reagent and then co-incubated at room temperature (RT) for an additional $10 \mathrm{~min}$. Absorbance was spectrophotometrically measured at $540 \mathrm{~nm}$, and NO concentration was calculated by extrapolation from a standard curve of sodium nitrite $\left(\mathrm{NaNO}_{2}\right)$.

\section{Cell apoptosis assays}

Neurons were incubated with or without BMSCs-Exos (100 $\mu \mathrm{g} /$ $\mathrm{mL})$ after treatment with glutamate (GLU; $100 \mu \mathrm{M})$ for $24 \mathrm{~h}$. A terminal deoxynucleotidyl transferase-mediated dUTP nick end labeling assay (TUNEL; Roche, Basel, Switzerland) was used to detect DNA strand breaks, according to the manufacturer's instructions. Images were taken by fluorescence microscope (AXIO Vert.A1 \& Imager A2; Carl Zeiss Microscopy GmbH, Jena, Germany). Apoptotic neuronal cells were determined by counting the number of total TUNEL- and 4',6-diamidino-2-phenylindole (DAPI)-stained neurons.

Flow cytometry was performed to verify the in vitro result of fluorescence microscopy. After incubation with GLU and BMSCsExos at 0 or $100 \mu \mathrm{g} / \mathrm{mL}$, respectively, neuronal cells were rinsed twice with PBS. Harvested cells were resuspended in binding buffer containing fluorescein isothiocyanate (FITC)-labeled Annexin V ( $5 \mu \mathrm{L}$; BD Biosciences) and propidium iodide (PI; $5 \mu \mathrm{L})$ for $5 \mathrm{~min}$ at RT in the dark. After washing three times with PBS, cells were immediately analyzed using a flow cytometer (FACSCalibur; BD Biosciences). The percentage of cells undergoing apoptosis was determined by dual-color analysis. 
A

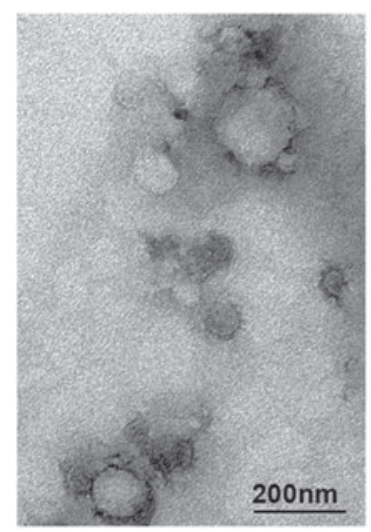

B

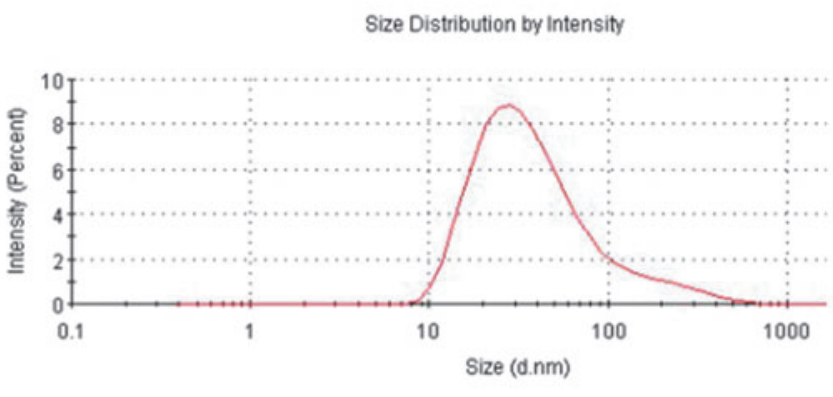

C

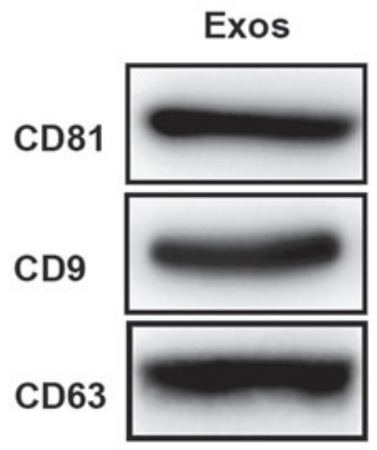

FIG. 2. Characterization of BMSCs-Exos. (A) Morphology observed by TEM. (B) Particle size distribution measured by DLS. (C) Western blot analysis of the specific exosome surface markers. BMSCs-Exos, bone mesenchymal stem cells/exosomes; DLS, dynamic light scattering; SCI, spinal cord injury; TEM, transmission electron microscopy. Color image is available online at www.liebertpub.com/neu

\section{Western blot analysis}

Protein lysates were extracted from cells and tissues (5-mm block of injured spinal cord tissue encompassing the lesion at day 1 or day 28 after SCI) by incubating with radioimmunoprecipitation assay lysis and extraction buffer (KeyGEN Biotechnology, Nanjing, China). Protein concentration was determined using BCA. Equal amounts of proteins were separated by sodium dodecyl sulfate polyacrylamide gel electrophoresis, transferred to polyvinylidene difluoride membranes (EMD Millipore Corp., Burlington, MA), and incubated overnight at $4^{\circ} \mathrm{C}$ with primary antibodies followed by blocking with bovine serum albumin $(5 \%$, v/v). Primary antibodies included cleaved caspase-3 (1:1000, rabbit IgG; Cell Signaling Technology, Danvers, MA), cleaved caspase-9 (1:1000, rabbit IgG; Cell Signaling Technology), B-cell lymphoma 2 (Bcl-2; 1:1000, rabbit IgG; Abcam), Bcl-2-associated X protein (Bax; 1:1000, rabbit IgG; Abcam), glyceraldehyde 3-phosphate dehydrogenase (GAPDH; 1:1000, rabbit IgG; Abcam), neurofilament $200 \mathrm{kDa}$ (NF200; 1:1000, mouse IgG1; Abcam), tumor necrosis factor alpha (TNF- $\alpha$; 1:1000, mouse IgG1; Abcam), interleukin (IL)-1 $\beta$ (1:1000, mouse IgG; Abcam), IL-6 (1:1000, mouse IgG; Abcam), and vascular endothelial growth factor (VEGF; 1:1000, rabbit IgG; Abcam). Membranes were then incubated with the secondary antibody (1:2000; Thermo Fisher Scientific) for $120 \mathrm{~min}$ at RT. Reacting bands were visualized using enhanced chemiluminescence reagent (Thermo Fisher Scientific), and density of the protein bands was semi-quantified using the software Image J (NIH, Bethesda, MD).

\section{Preparation of contusive spinal cord injury rat model and experimental groups}

All procedures were conducted according to the Guidelines for the Care and Use of Laboratory Animals and approved by the
Animal Care and Use Committee of Nanjing Medical University. Female rats (170-220 g) were anesthetized with chloral hydrate ( $350 \mathrm{mg} / \mathrm{kg}$ of body weight). After animals were unresponsive, skin and muscle were opened on the back to expose the vertebral column. A T10 laminectomy was performed and the exposed dorsal surface of the cord was subjected to weight-drop impact using a 10$\mathrm{g}$ rod (2.5 mm in diameter; C4p01-001; RWD Life Science Corp., Shenzhen, China) dropped from a height of $12.5 \mathrm{~mm}$. Muscles were sutured immediately after administration, and the skin was then closed. Bladders of animals were manually voided three times per day until reflexive control of bladder function was restored.

Rats were randomly assigned into two groups: SCI or Exos ( $n=10$ /group). Rats were subjected to SCI, followed by tail vein injection of BMSCs-Exos (200 $\mu \mathrm{g}$ of total protein of BMSCs-Exos precipitated in $200 \mu \mathrm{L}$ of PBS) or an equal volume of PBS $(200 \mu \mathrm{L})$ immediately after SCI.

\section{Assessment of locomotor capacity}

Locomotion recovery after SCI was scored according to the Basso, Beattie, and Bresnahan (BBB) open field 21-point locomotion rating scale. ${ }^{36}$ Movement of hindlimbs was assessed weekly by two independent examiners blinded to the treatment regimen.

\section{Magnetic resonance imaging}

Three animals in each group were randomly selected for magnetic resonance imaging (MRI) examination at day 1 . Rats were anesthetized with halothane (3-4\% induction, $1.5-2 \%$ maintenance) in oxygen $(0.4 \mathrm{~L} / \mathrm{min})$ and nitrogen $(0.6 \mathrm{~L} / \mathrm{min})$. Anesthetized rats were placed on a fixation system in a prone position. Experiments were performed on a small animal MRI system (Bruker BioSpec 7T/20 USR; Bruker AXS GmbH, Karlsruhe, Germany). The sequence protocol was performed with the following parameters:

FIG. 3. Functional influence of BMSCs-Exos on HUVECs and microglia. (A) BMSCs-Exos significantly enhanced the proliferation of HUVECs when analyzed by CCK-8 assay. (B) BMSCs-Exos significantly reduced NO production in microglia when treated with LPS. (C) Tubule formation assay. BMSCs-Exos significantly enhanced tube formation ability compared with the PBS group at $6 \mathrm{~h}$. Scar bar, $100 \mu \mathrm{m}$. (D) Quantitative analysis of tubule formation assay. The values of total branching points and total tube length were all significantly higher in the BMSCs-Exos group than in the PBS group. (E) Transwell assay. Representative photomicrographs showed the effect of BMSCs-Exos on the migration of HUVECs after incubation for $24 \mathrm{~h}$. Scar bar: $100 \mu \mathrm{m}$. (F) Quantitative analysis of the transwell assay. (G) Scratch wound assay. BMSCs-Exos significantly increased the migration ability of HUVECs at 12 and $24 \mathrm{~h}$. Scar bar, $250 \mu \mathrm{m}$. (H) Quantitative analysis of the migration rate of HUVECs. *Statistically significant difference compared with the PBS group $(p<0.05) ;{ }^{\#} p<0.05$ between LPS and BMSCs-Exos groups. BMSCs-Exos, bone mesenchymal stem cells/exosomes; CCK-8, Cell Counting Kit-8; HUVECs, human umbilical vein endothelial cells; LPS, lipopolysaccharide; NO, nitric oxide; OD, optical density; PBS, phosphate-buffered saline. Color image is available online at www.liebertpub.com/neu 


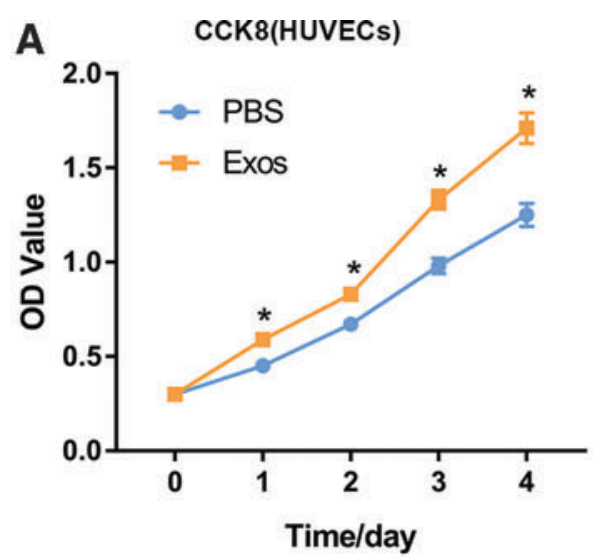

C

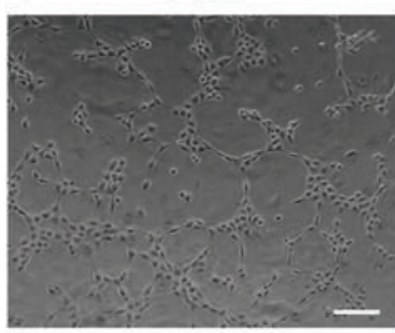

Exos

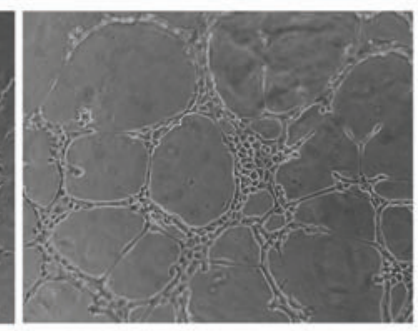

E

PBS

Exos

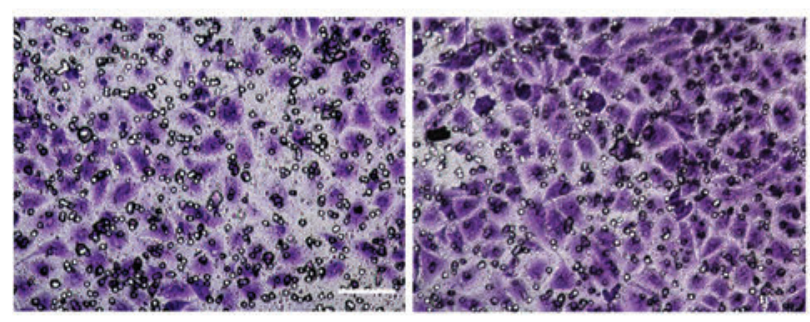

G
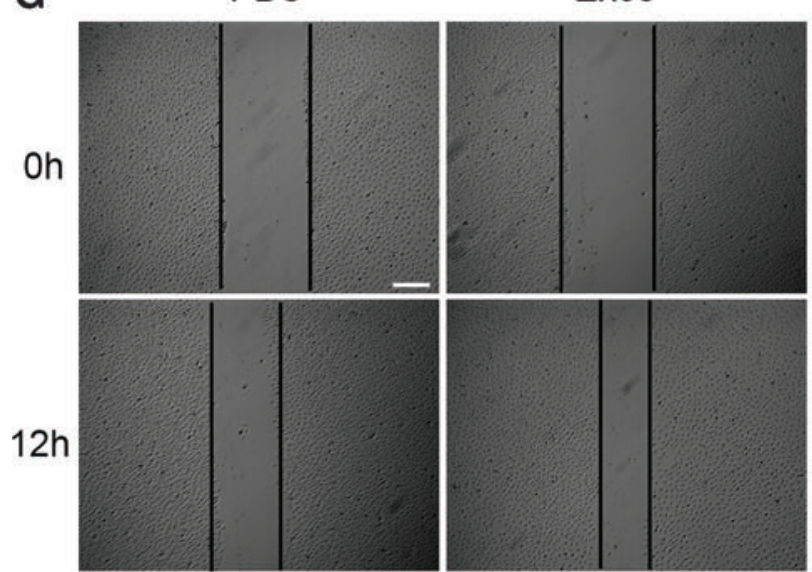

$24 \mathrm{~h}$

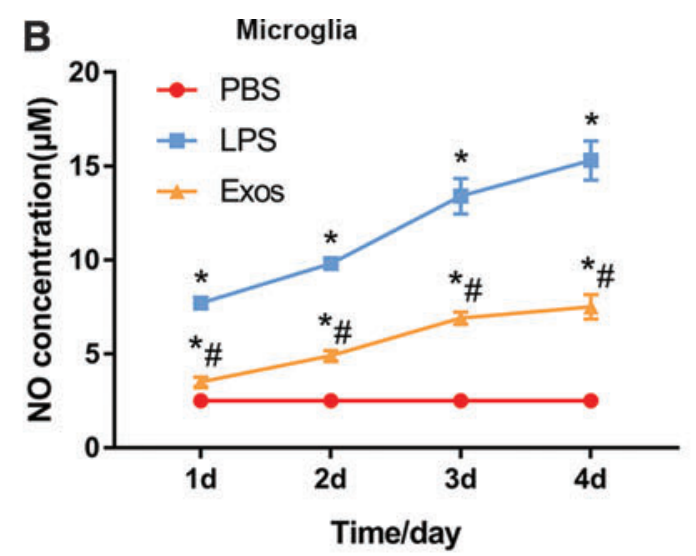

D

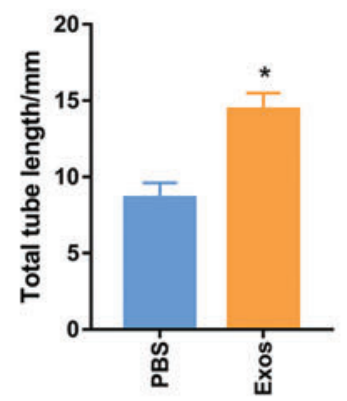

F
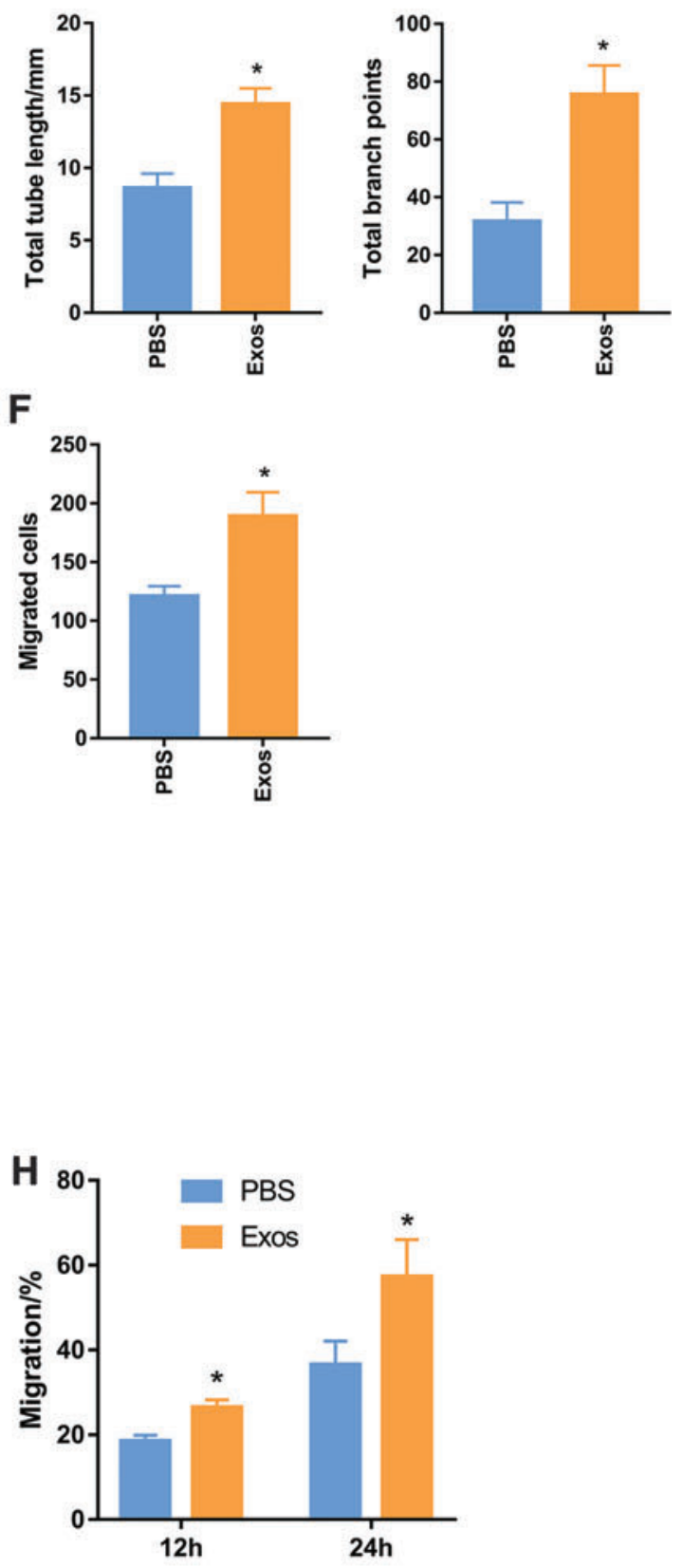
T2-weighted; $256 \times 256$ matrix; slice thickness, $1 \mathrm{~mm}$; intersection gap, $1 \mathrm{~mm}$; echo time/repetition time: $27 / 3000 \mathrm{~ms}$; rapid acquisition with relaxation enhancement factor, 16; and flip angle, 90 degrees. T2-weighted images were acquired in the sagittal and axial planes by ParaVision (version 6.0.1, Bruker BioSpec; Bruker AXS $\mathrm{GmbH})$.

\section{Tissue processing}

Animals were anesthetized with a lethal dose of chloral hydrate at day 1 and day 28 post-injury. Ice-cold physiological saline was perfused transcardially followed by ice-cold paraformaldehyde (PFA; 4\%, w/v). Afterward, segments of injured spinal cord containing the lesion site were acquired carefully, fixed in PFA (4\%, w/ v) for $24 \mathrm{~h}$ at $4^{\circ} \mathrm{C}$, and transferred to sucrose $(20 \%$, w/v) and sucrose $(30 \%, w / v)$ PBS solution. Spinal cord tissues were then embedded in optimum cutting temperature compound and cut into serial longitudinal sections with a thickness of approximately $18 \mu \mathrm{m}$. All slides were stored at $-80^{\circ} \mathrm{C}$ until further use.

\section{In situ apoptotic assay terminal deoxynucleotidyl transferase-mediated dUTP nick end labeling assay staining}

Apoptotic cells in the spinal cord at day 1 post-injury were identified and quantified by TUNEL (Promega), according to the manufacturer's protocols. Images were captured, and apoptotic cell nuclei were characterized with green fluorescence.

\section{Histopathological analyses}

Nissl substance in the cytoplasm of neurons from spinal cord sections of each group were stained at day 28 after injury with cresyl violet (FD NeuroTechnologies, Columbia, MD). Spinal cord sections were rinsed in distilled water and subsequently stained in cresyl violet solution for $10 \mathrm{~min}$. After rinsing with distilled water, sections were differentiated in $95 \%$ ethyl alcohol, cleared with xylene, and mounted with neutral balsam. Severe disruption of tissue organization and/or the loss of staining were identified as traumatic lesion areas. The identified areas in each section were measured by ImageJ software (NIH). Lesion volume was obtained by the sum of the total lesion area multiplied by the distance (approximately $200 \mu \mathrm{m}$ ) between the sections.

\section{Spinal cord tissue immunofluorescence}

Primary antibodies used in this study included NF200 (1:50, mouse IgG1; Abcam, Cambridge, UK), glial fibrillary acidic protein (GFAP; 1:1000, rabbit IgG1; Abcam), CD68 (1:200, mouse IgG1; EMD Millipore Corp.), CS56 (1:350, mouse IgM; Abcam), CD31 (1:200, mouse IgG1; Abcam), and C3 (1:200, mouse IgG; Abcam). The secondary antibody was a cyanine 3- or FITCconjugated secondary antibody (1:200; Jackson ImmunoResearch, West Grove, PA). First, spinal cord sections at day 1 or 28 postinjury were permeabilized in Triton X-100 PBS solution $(0.3 \%$, w/ v) for $30 \mathrm{~min}$ and then blocked with natural goat serum PBS solution $(10 \%, v / v)$. Specimens were incubated with primary antibodies overnight at $4^{\circ} \mathrm{C}$, triple washed with PBS, and incubated with secondary antibody for $2 \mathrm{~h}$ at RT. Nuclei were then stained with DAPI, and fluorescent images were taken. For each slide, the lesion was identified as the area lacking staining. We selected six different areas near the traumatic lesion as the near-injury area. Six different areas at least $10 \mathrm{~mm}$ in distance from the traumatic lesion were chosen as far-injury areas. Average intensity of NF200 and CS56 was measured with ZEN lite software. Data are expressed as the percentage of intensity increase or decrease in the near-injury area compared with the far-injury area. For $\mathrm{CD}^{+} 8^{+}$cell counting, $\mathrm{CD} 68^{+}$immunostained images were captured at lesion interfaces and traumatic lesion sites. A1 neurotoxic reactive astrocytes were identified as C3-positive astrocytes. All images were taken at the same exposure time and conditions.

\section{Statistical analyses}

Data are expressed as mean \pm standard deviation for at least three independent experiments. Two-group comparisons were tested with an independent Student's $t$-test. Repeated-measures analysis of variance followed by Bonferroni post-hoc corrections were performed for comparsions of BBB scores. Asterisk (*) and pound sign (\#) represented a $p$ value $<0.05$.

\section{Results}

\section{Characterization of bone mesenchymal stem cells/ exosomes}

Analyses, including TEM, dynamic light scattering (DLS) analysis, and western blot, were performed in order to comprehensively characterize the purified nanoparticles derived from BMSCs. As shown in Figure 2A, BMSCs-Exos exhibited a roundshaped morphology under TEM, indicating the presence of exosomes. DLS analysis revealed that the diameter size distribution of these nanoparticles varied from 20 to $150 \mathrm{~nm}$ (Fig. 2B), which was similar to previous reports. Moreover, specific exosome surface markers, including $\mathrm{CD} 9, \mathrm{CD} 63$, and $\mathrm{CD} 81$, were positive in BMSCs-Exos according to western blotting results (Fig. 2C), which further confirmed the presence of exosomes. All analyses presented above indicated that the exosomes derived from BMSCs were successfully isolated and identified.

\section{Bone mesenchymal stem cells/exosomes promote proliferation, migration, and tube formation of human umbilical vein endothelial cells}

To explore the potential functional roles of BMSCs-Exos in cell proliferation, HUVECs were cultured with BMSCs-Exos at a concentration of $100 \mu \mathrm{g} / \mathrm{mL}$ or without BMSCs-Exos for a series of continued functional experiments. Proliferation of HUVECs was examined by a CCK-8 assay (Fig. 3A). The result showed that incubation with BMSC-Exos at a concentration of $100 \mu \mathrm{g} / \mathrm{mL}$ resulted in a remarkable increase in proliferation of HUVECs.

Tubule lengths and branch points were counted after HUVECs began forming capillary tubes. As shown in Figure 3C,D, BMSCsExos $(100 \mu \mathrm{g} / \mathrm{mL})$ significantly enhanced tube formation at $6 \mathrm{~h}$ compared to the PBS group. Transwell assay showed that incubation with BMSCs-Exos significantly promoted HUVECs migration (Fig. 3E,F). Scratch wound assays were utilized to determine the effect of BMSCs-Exos on HUVECs migration. The result showed that application of exosomes remarkably increased the motility ability of HUVECs (Fig. 3G,H).

\section{Effect of bone mesenchymal stem cells/exosomes on lipopolysaccharide-induced nitric oxide production}

NO production was extremely low in the PBS group whereas treatment with LPS $(5 \mathrm{ng} / \mathrm{mL})$ resulted in a significant increase of NO production. Pre-treatment with BMSC-Exos $(100 \mu \mathrm{g} / \mathrm{mL})$ remarkably decreased LPS-induced NO production at 24, 48, 72, and $96 \mathrm{~h}$ compared to the LPS group, but was still significantly higher than in the PBS group (Fig. 3B). 

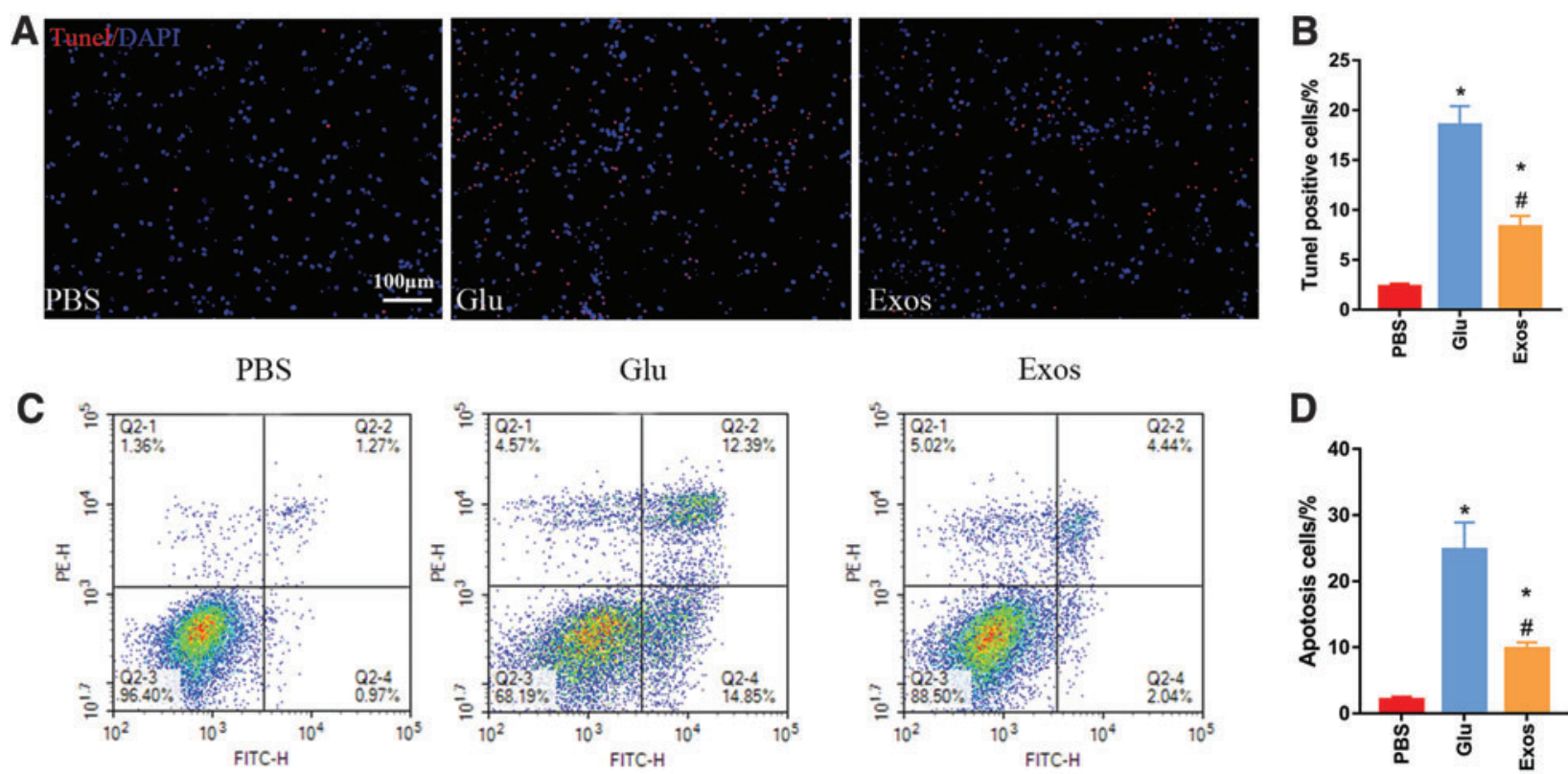

$\mathbf{E}$
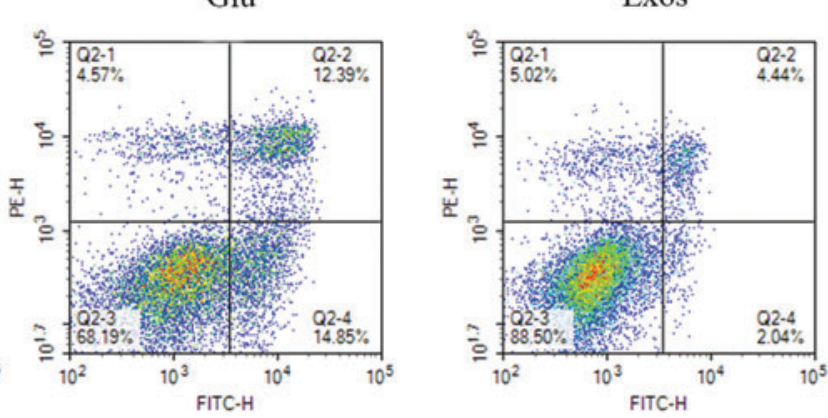

D

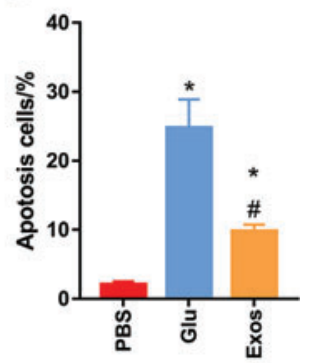

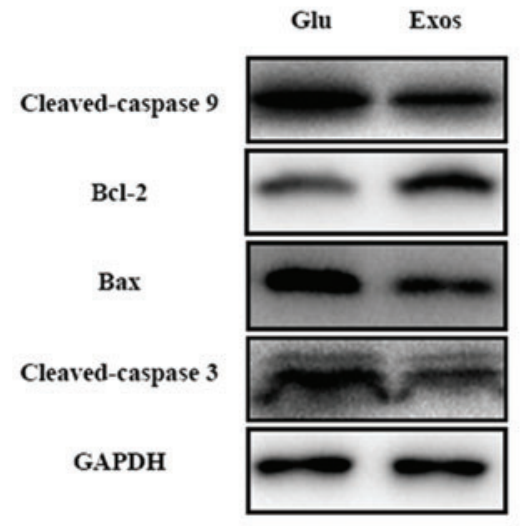

$\mathbf{F}$

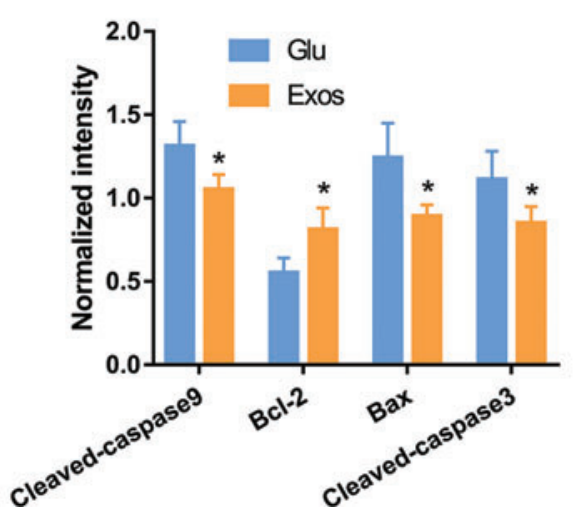

FIG. 4. BMSCs-Exos alleviates GLU-induced neuronal apoptosis in vitro. (A) TUNEL assay. Representative fluorescence images of TUNEL-positive apoptotic cells (red) induced by GLU together with or without BMSCs-Exos administration. Cell nuclei were stained with DAPI (blue) (B) Comparison of the number of TUNEL-positive cells with and without BMSCs-Exos treatment. $* p<0.05$ compared to the PBS group; ${ }^{\#} p<0.05$ between GLU and BMSCs-Exos groups. (C) Neuronal apoptosis induced by GLU together with or without BMSCs-Exos administration was assessed through Annexin V/FITC/PI double staining with flow cytometric analysis. (D) Quantitative results of apoptotic neurons with and without treatment by BMSCs-Exos. $* P<0.05$ compared with the PBS group; ${ }^{\#} p<0.05$ between GLU and BMSCs-Exos groups. (E) Western blot analysis of apoptosis-related proteins in neurons incubated with BMSCs-Exos treatment after GLU-induced excitotoxicity. (F) Semiquantification of relative expression levels of apoptosis-related proteins in primary neurons normalized to GAPDH. * $p<0.05$ compared to the GLU group. Bax, Bcl-2-associated X protein; Bcl-2, Bcell lymphoma 2; BMSCs-Exos, bone mesenchymal stem cells/exosomes; FITC, fluorescein isothiocyanate; GAPDH, glyceraldehyde 3phosphate dehydrogenase; GLU, glutamate; PI, propidium iodide; TUNEL, terminal deoxynucleotidyl transferase-mediated dUTP nick end labeling assay. Color image is available online at www.liebertpub.com/neu

\section{Bone mesenchymal stem cells/exosomes reduce neuronal cell death in vitro}

High levels of GLU around the synaptic cleft may result in neuronal cell death and represent one of the most important pathological hallmarks of SCI. ${ }^{37}$ Neuronal apoptosis induced by traumatic injury to the spinal cord contributes to neuronal cell death and results in neurological dysfunction in rats. ${ }^{38}$ Accordingly, we used TUNEL to study neuronal apoptosis (Fig. 4A). As shown in Figure 4A,B, after administration of GLU, neurons exhibited a relative ratio of apoptosis. We then evaluated the neuroprotective effect of BMSCs-Exos in a GLU-induced excitotoxicity model. The result demonstrated that the number of TUNEL-positive neuronal cells decreased remarkably after BMSCs-Exos application $(100 \mu \mathrm{g} / \mathrm{mL})$, indicating a neuroprotective effect of BMSCs-Exos on GLU-induced excitotoxicity.

Next, we continued to assess cell apoptosis using Annexin VFITC/PI double staining with flow cytometry, where the early and late apoptosis rate of cells was measured. As shown in Figure 4C,D, exposure to GLU dramatically increased apoptosis compared to the control group whereas administration of BMSCs-Exos significantly attenuated the apoptotic effect of GLU in neuronal cells.

Western blotting results demonstrated that the level of proapoptotic proteins, including Bax, activated caspase-3, and caspase9, decreased upon co-treatment with BMSCs-Exos after 24-h 
A

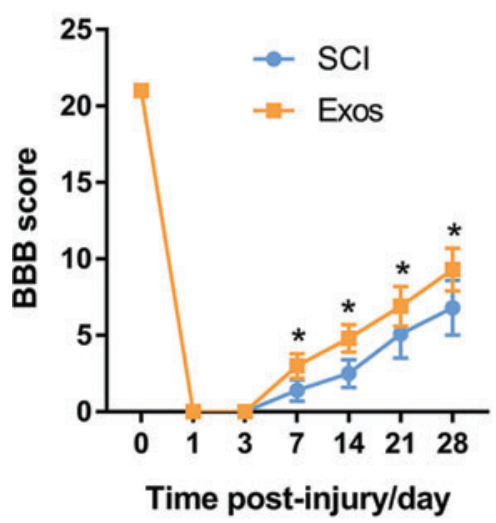

B

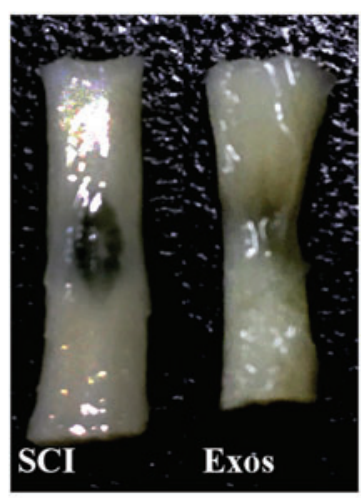

C
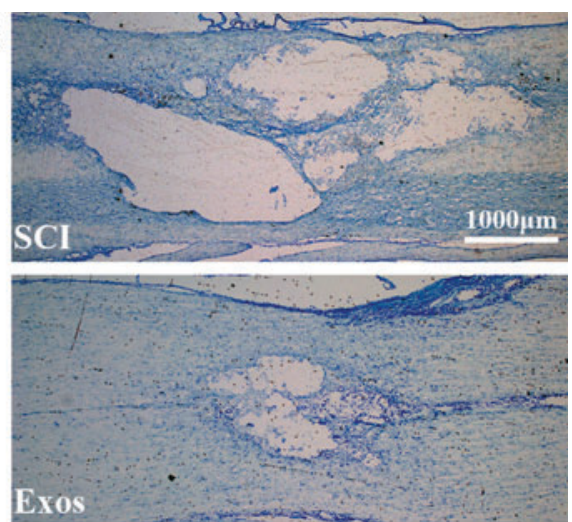

A
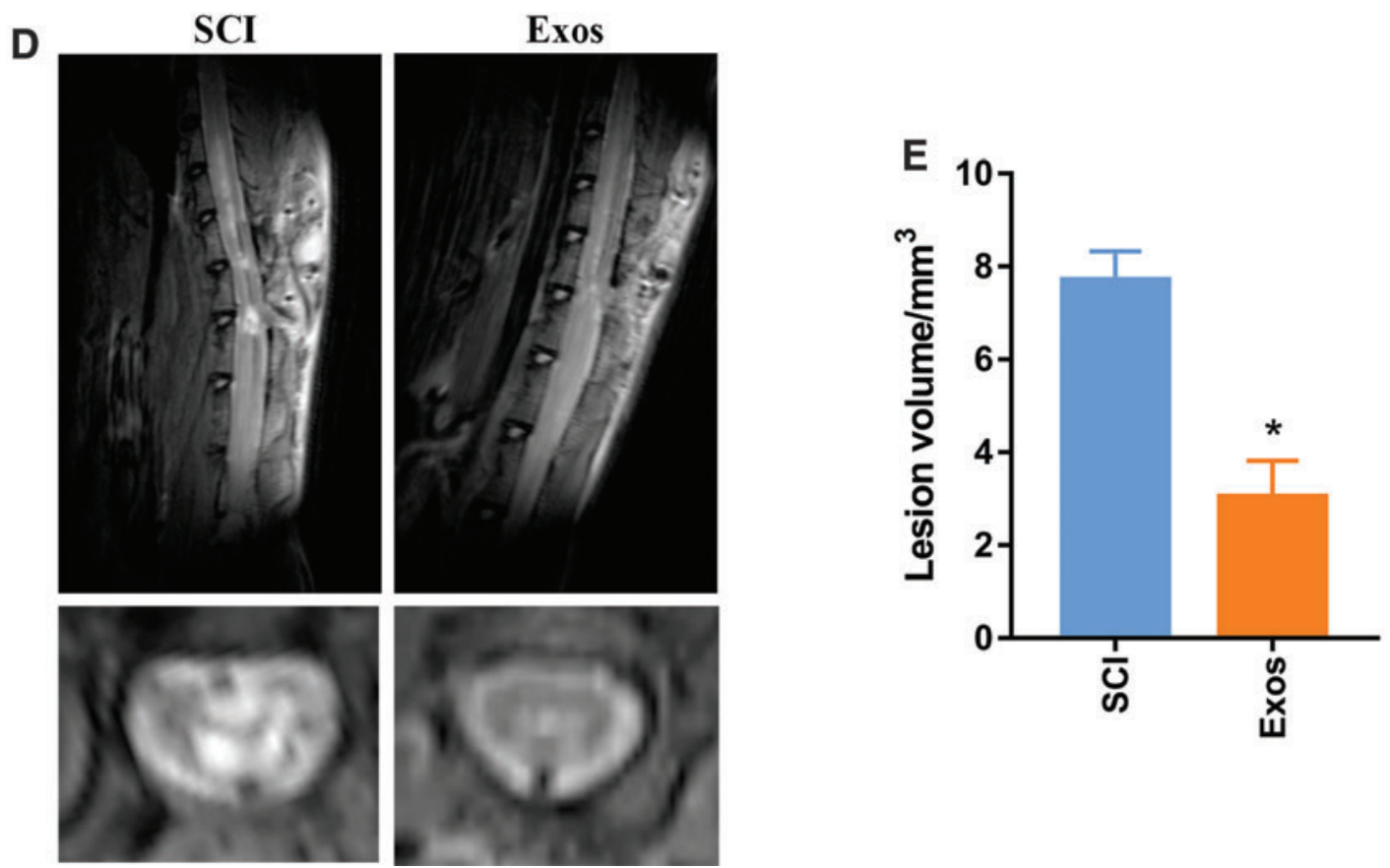

FIG. 5. BMSCs-Exos promoted functional behavioral recovery and reduced lesion tissue area after SCI. (A) BBB grading scale was used to functionally grade the rats in both groups up to 28 days post-injury. (B-E) Spinal cord at day 28 post-injury. (B) Gross morphology. (C) Representative Nissl-stained sagittal sections. (D) Representative sagittal and coronal MRI images. (E) Quantitative analysis of the lesion volumes compared between SCI and BMSCs-Exos groups. * $p<0.05$ compared to the SCI group. BBB, Basso, Beattie, and Bresnahan; BMSCs-Exos, bone mesenchymal stem cells/exosomes; MRI, magnetic resonance imaging; SCI, spinal cord injury. Color image is available online at www.liebertpub.com/neu

exposure to GLU, whereas the level of the antiapoptotic protein, Bcl-2, increased after incubation with BMSCs-Exos (Fig. 4E,F).

These data indicated that BMSCs-Exos could protect neurons from GLU-induced apoptosis in vitro.

\section{Bone mesenchymal stem cells/exosomes reduce lesion volume of traumatic spinal cord injury}

In order to verify the neuroprotective effect of BMSCs-Exos in vivo, we performed MRI and assessed motor function and histological injury in SD rats that underwent a weight-drop contusive injury at the site of thoracic spinal cord (T10). After traumatic SCI, motor behavior was evaluated by the 21-point BBB locomotor rating scale in an open field. Results showed that total hindlimb paralysis $(\mathrm{BBB}$ score $=0$ ) was found in both groups at days 1 and 3 post-injury. However, rats in the BMSCs-Exos group exhibited significant improvement in the BBB score compared to rats in the SCI group from the first week post-trauma until sacrifice in the fourth week (Fig. 5A).

As shown in Figure 5B, the traumatic lesion site in the injured spinal cord was clearly visible in the gross morphology of the injured spinal cords. After treatment with BMSCs-Exos, the lesion area was notably smaller than in the SCI group. Next, we identified the traumatic lesion cavity by loss of cells using Nissl staining to study whether BMSCs-Exos administration could reduce the lesion volume significantly. Four weeks after SCI, dramatic SCI tissue loss was observed. However, administration of BMSCs-Exos significantly reduced the area of tissue loss post-traumatically (Fig. 5C,E).

Randomly selected rats from each group at day 28 after SCI were analyzed by 7.0 Tesla MRI (BioSpec 70/20USR; Bruker). MRI demonstrated that injection with BMSC-Exos remarkably reduced the lesion area compared to the SCI group (Fig. 5D). 

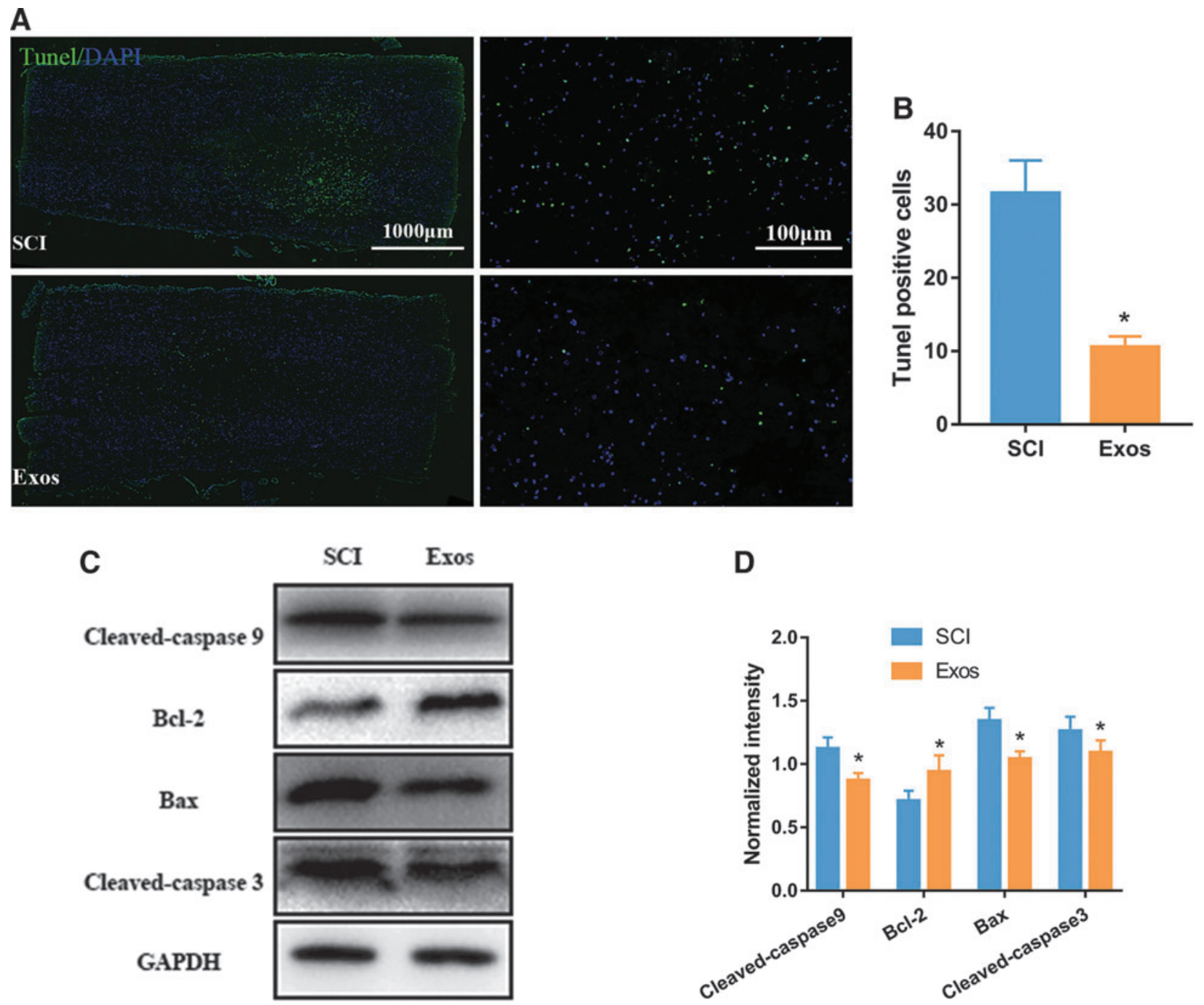

FIG. 6. BMSCs-Exos protects injured neurons from apoptosis in vivo. (A) Representative images of TUNEL-positive apoptotic cells (green) in sagittal spinal cord sections at day 1 post-injury. Nuclei of all cells were stained with DAPI (blue). (B) Comparison of the number of TUNEL-positive cells with and without BMSCs-Exos treatment. (C) Western blots of apoptosis-associated proteins in lesion extracts at day 1 post-injury. (D) Semiquantification of relative expression level of apoptosis indicated proteins, normalized to GAPDH. Level of significance was set at $* p<0.05$. Bax, Bcl-2-associated X protein; Bcl-2, B-cell lymphoma 2; BMSCs-Exos, bone mesenchymal stem cells/ exosomes; DAPI, 4',6-diamidino-2-phenylindole; GAPDH, glyceraldehyde 3-phosphate dehydrogenase; SCI, spinal cord injury; TUNEL, terminal deoxynucleotidyl transferase-mediated dUTP nick end labeling assay. Color image is available online at www.liebertpub.com/neu

All of these data suggest that BMSCs-Exos administration effectively reduced traumatic SCI lesion volume.

\section{Bone mesenchymal stem cells/exosomes attenuates neuronal cell death in spinal cord injury in vivo}

TUNEL assays were applied to assess neuronal cell apoptosis in the traumatic area of the spinal cord in vivo. On day 1 post-injury, a dramatic reduction in the number of TUNEL-positive (apoptotic) cells was found in the BMSCs-Exos group compared to the SCI group (Fig. 6A,B). In vivo TUNEL assays further confirmed that BMSCs-Exos effectively protected cells from apoptosis after traumatic SCI, which was consistent with in vitro results.

According to western blot results, expression level of apoptosisassociated markers, including Bax, cleaved-caspase-3, and cleaved-caspase-9, was significantly reduced in the BMSCs-Exos group compared to the SCI group, whereas expression level of the antiapoptotic protein, Bcl-2, was significantly enhanced, which further confirmed our in vitro results (Fig. 6C,D).

\section{Bone mesenchymal stem cells/exosomes reduce glial scar formation and promoted angiogenesis after spinal cord injury}

Many studies showed that the formation of a glial scar is an impediment for axonal regeneration, and axons usually stop at the border of the glia-pial barrier. Therefore, axons transverse the scar barrier in the injured site to promote functional recovery. By staining with CS56 to represent CSPGs expressed by reactive astrocytes, we found that CSPG deposition was significantly reduced in the lesion area of rats in the BMSCs-Exos group at day 28 post-injury (Fig. 7A,B).

Vascularization of newly formed tissues plays an important role in the injured spinal cord healing process. ${ }^{39}$ Newly formed vessels at injured spinal cord sites were characterized by CD31 staining and 

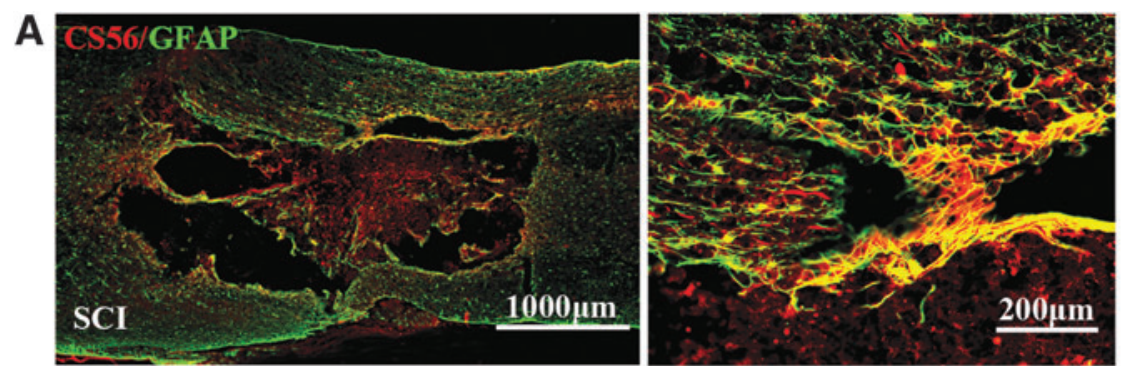

B
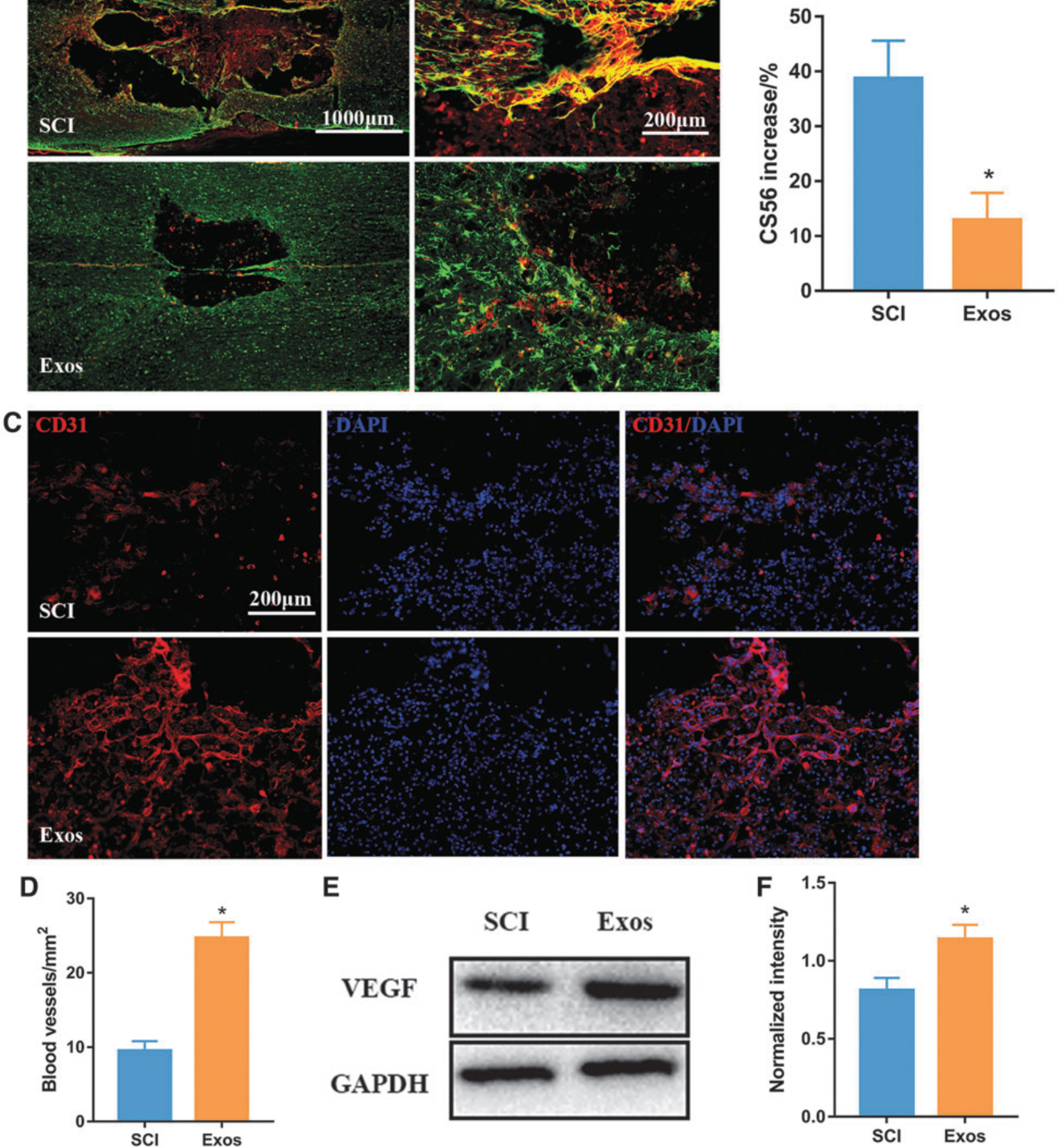

FIG. 7. BMSCs-Exos reduces deposition of CSPGs and promotes angiogenesis after SCI. (A) Representative immunohistochemical staining images of CS56 (red) and GFAP (green) in the spinal cord tissues of SCI and BMSCs-Exos groups at day 28 post-injury. Nuclei of all cells were stained with DAPI (blue). (B) Semiquantification of CS56 intensity increase. (C) Representative immunohistochemical staining images of CD31 (red) in spinal cord tissue of SCI and BMSCs-Exos groups at day 28 post-injury. Nuclei of all cells were stained with DAPI (blue). (D) Quantitative analysis of the total number of blood vessels. (E) Representative western blots of VEGF expression levels in lesion extracts at day 28 post-trauma. (F) Semiquantification of relative expression level of VEGF protein, normalized to GAPDH. The level of significance was set at * $p<0.05$. BMSCs-Exos, bone mesenchymal stem cells/exosomes; CSPGs, chondroitin sulfate proteoglycans; DAPI, 4',6-diamidino-2-phenylindole; GAPDH, glyceraldehyde 3-phosphate dehydrogenase; GFAP, glial fibrillary acidic protein; SCI, spinal cord injury; VEGF, vascular endothelial growth factor. Color image is available online at www.liebertpub.com/neu 

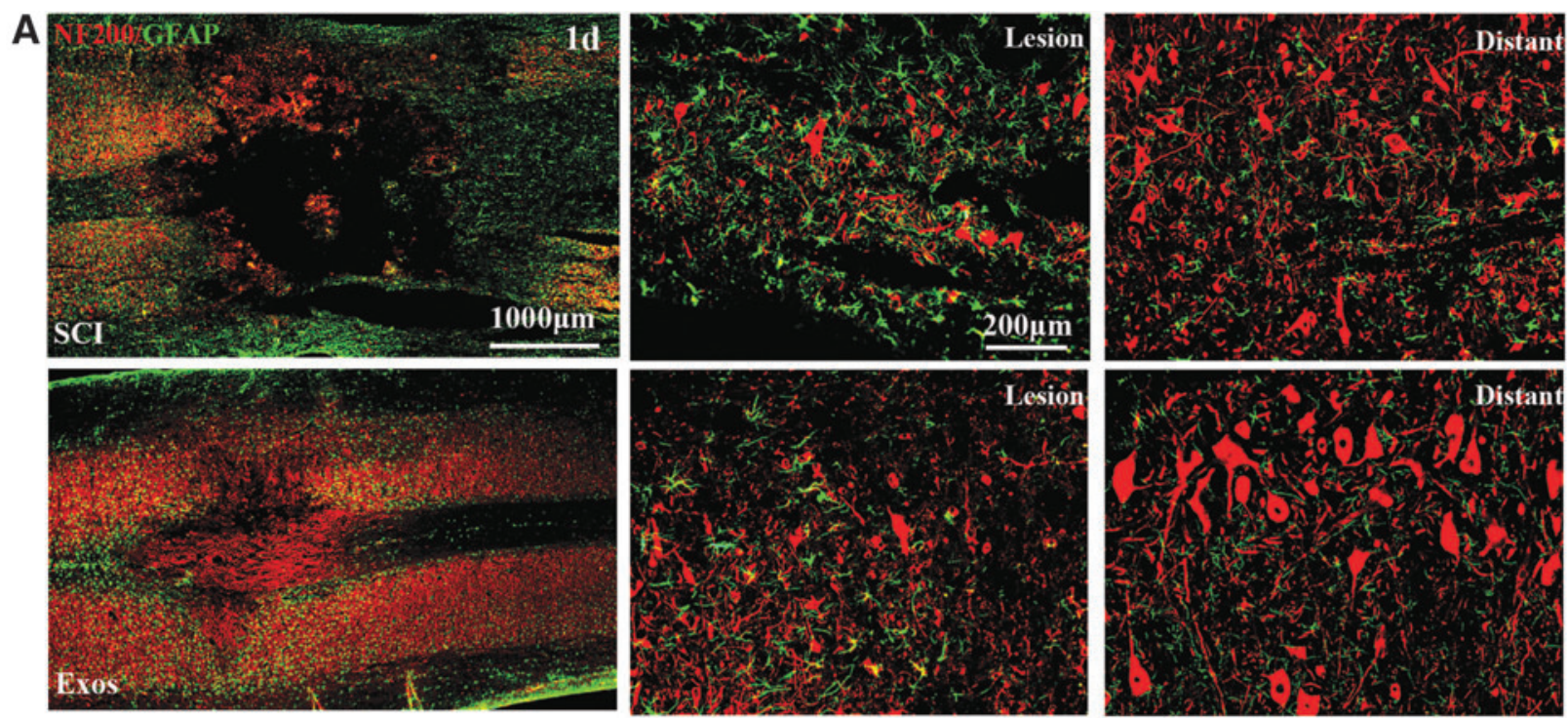

B
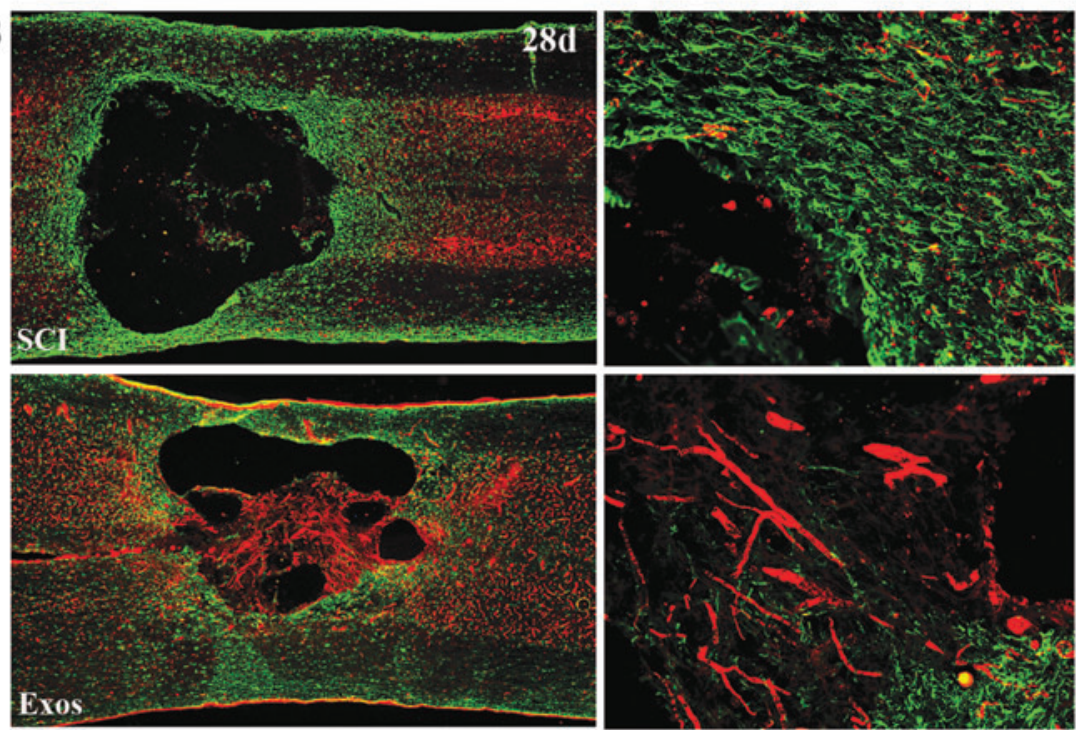

C

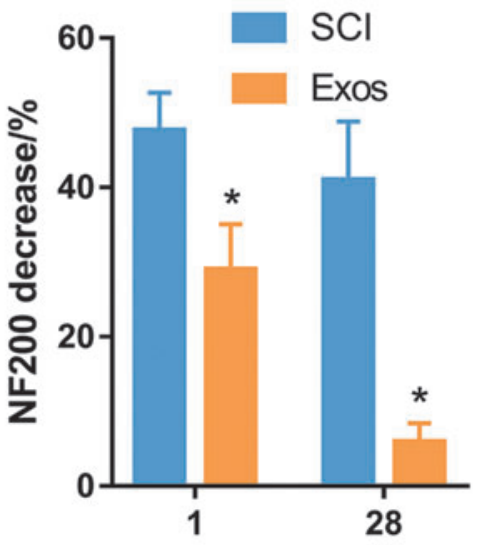

Days post-injury

FIG. 8. BMSCs-Exos promote axonal regeneration in the injured lesion area of the spinal cord. (A and B) Representative immunohistochemical staining images of NF200 (red) and GFAP (green) in the injured spinal cord tissues of SCI and BMSCs-Exos groups at day 1 (A) and day 28 (B) post-injury. (C) Semiquantification of NF200 intensity decrease. Level of significance was set at $* p<0.05$. BMSCs-Exos, bone mesenchymal stem cells/exosomes; GFAP, glial fibrillary acidic protein; NF200, neurofilament 200 kDa; SCI, spinal cord injury. Color image is available online at www.liebertpub.com/neu

average vessel densities were quantified afterward. As shown in Figure 7C,D, newly formed vessels were remarkable more abundant in the BMSCs-Exos group compared to the SCI group at day 28 post-injury. Moreover, western blot results revealed that VEGF expression level in the BMSCs-Exos group was significantly upregulated compared to the SCI group at day 28 post-injury (Fig. 7E,F). These data showed that the number of newly formed vessels increased in the injured lesion of the spinal cord in the BMSCs-Exos group compared to the SCI group at day 28 post-injury.

\section{Bone mesenchymal stem cells/exosomes promote axonal regeneration in the injured lesion area of the spinal cord}

We further examined the density or status of neurons and axons in the injured spinal cord in order to elucidate the anatomical basis of the observed locomotor recovery. Neurofilaments are cell-typespecific proteins in the central nervous system (CNS) that are regarded as potential surrogate markers of neuronal and axonal damage. ${ }^{40}$ Immunostaining analysis of the $200-\mathrm{kDa}$ subunit of a neurofilament (NF200), which contributes to anomalous electrophoretic mobility, has been used to evaluate neuronal and axonal damage. ${ }^{41}$ The decrease in the staining against NF200 in the lesion areas compared with the distant area, as assessed by average pixel intensity values, was much lower in the BMSCs-Exos group than in the SCI group at day 1 (Fig. 8A,C) and at day 28 post-injury (Fig. 8B,C), suggesting that administration of BMSCs-Exos promoted remarkable axonal regeneration after traumatic SCI. Western blot analysis of NF200 was also used to further confirm the immunofluorescence analysis (Fig. 9D). Expression level of NF200 was significantly higher in the BMSCs-Exos group than in the SCI group at day 1 post-injury. 

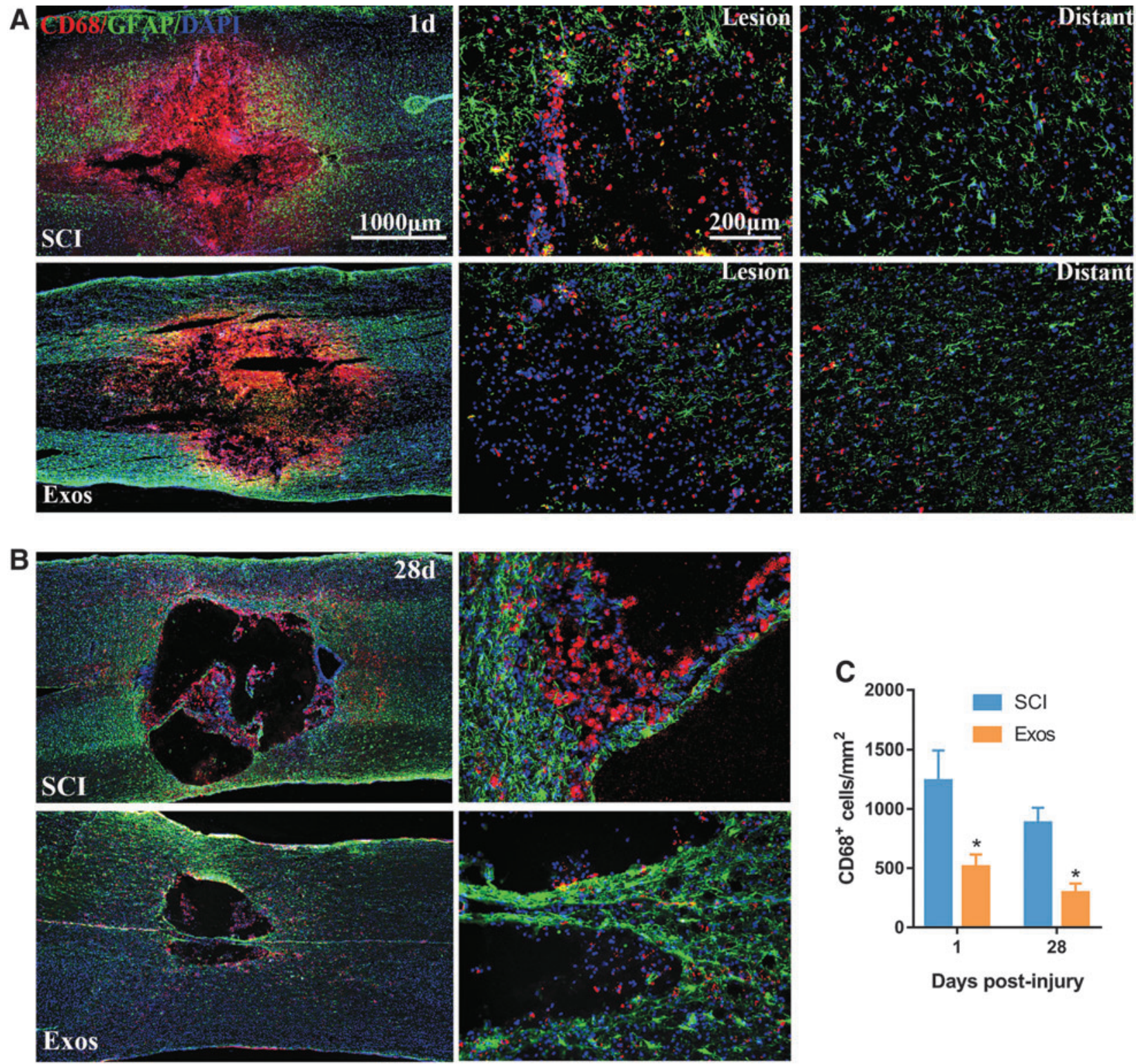

Days post-injury
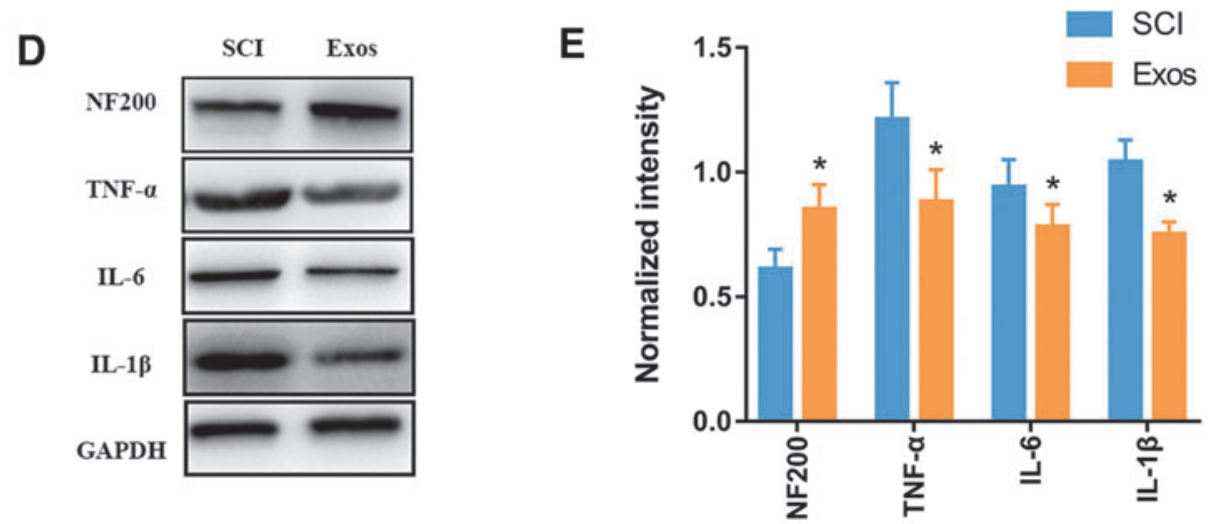

FIG. 9. BMSCs-Exos suppresses activation of microglia and reduces neuroinflammation after SCI. (A and B) Representative immunohistochemical staining images of CD68 (red) and GFAP (green) in injured spinal cord tissue of the SCI and BMSCs-Exos groups at day 1 (A) and day 28 (B) post-injury. Nuclei of all cells were stained with DAPI (blue). (C) Analysis of the number of CD68 ${ }^{+}$ microglia in the traumatic lesion area. (D) Representative western blots of inflammation-associated proteins in lesion extracts and NF200 at day 1 post-trauma. (E) Semiquantification of relative expression level of NF200, TNF- $\alpha$, IL-6, and IL-1 $\beta$ normalized to GAPDH. Level of significance was set at * $p<0.05$. BMSCs-Exos, bone mesenchymal stem cells/exosomes; DAPI, 4',6-diamidino-2-phenylindole; GAPDH, glyceraldehyde 3-phosphate dehydrogenase; GFAP, glial fibrillary acidic protein; IL, interleukin; NF200, neurofilament 200 kDa; SCI, spinal cord injury; TNF- $\alpha$, tumor necrosis factor alpha. Color image is available online at www.liebertpub.com/neu 

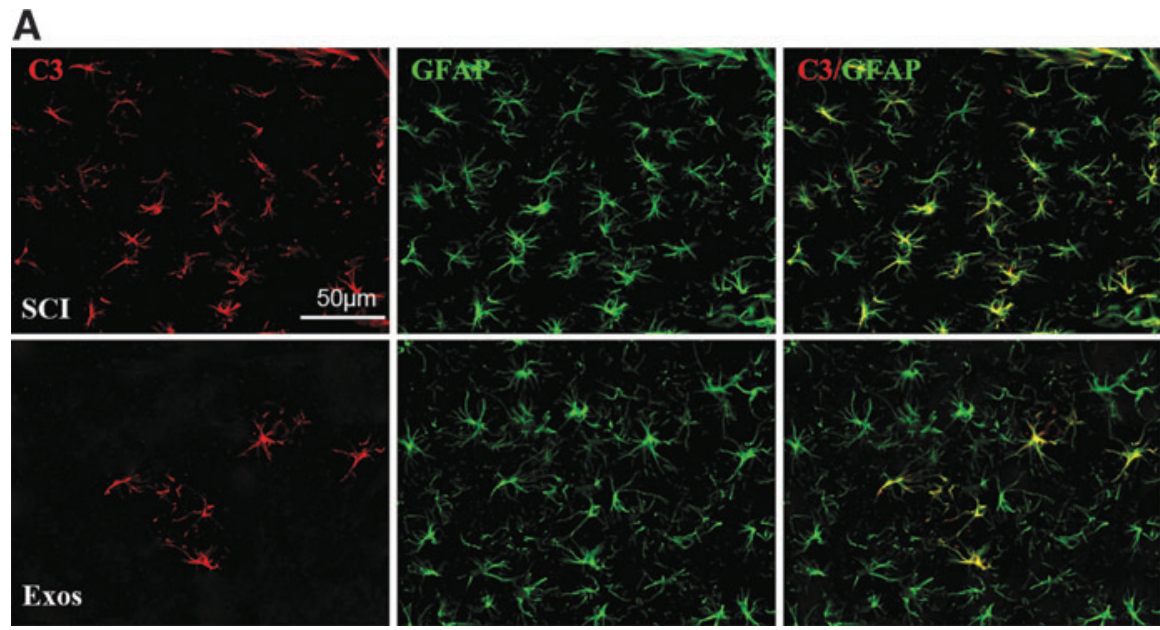

B

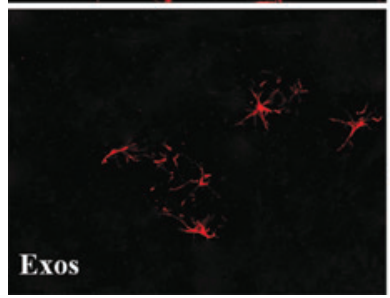

FIG. 10. BMSCs-Exos suppressed activation of A1 neurotoxic reactive astrocytes. (A) Representative immunohistochemical staining images of C3 (red) and GFAP (green) in the injured spinal cord tissues of SCI and BMSCs-Exos groups at day 1. (B) Quantitative analysis of the ratio of $\mathrm{C}^{+} / \mathrm{GFAP}^{+}$cells in the traumatic lesion area. BMSCs-Exos, bone mesenchymal stem cells/exosomes; GFAP, glial fibrillary acidic protein; SCI, spinal cord injury. Color image is available online at www.liebertpub.com/neu

\section{Bone mesenchymal stem cells/exosomes suppresses the activation of microglia and reduces neuroinflammation after spinal cord injury}

To evaluate the effect of BMSCs-Exos on microglial activation after SCI, we quantified the number of activated microglial cells adjacent to the injury lesion site by immunodetection of $\mathrm{CD}^{+} 8^{+}$ microglia. A significant reduction in the number of CD68-positive microglia in the traumatic lesion area was found in the BMSCsExos treatment group compared to the SCI group at both day 1 (Fig. 9A,C) and 28 post-injury (Fig. 9B,C). Moreover, we used western blot to further confirm these results. Expression levels of inflammation markers, including TNF- $\alpha$, IL- $1 \beta$, and IL-6, were remarkably reduced in the exosome treatment group compared to the SCI group (Fig. 9D,E). All these data showed that administration of BMSCs-Exos could reduce neuroinflammation after SCI.

\section{Bone mesenchymal stem cells/exosomes suppressed the activation of $A 1$ neurotoxic reactive astrocytes}

As previously reported, astrocytes could be divided into two different types, named A1 astrocytes and A2 astrocytes. ${ }^{42-45}$ Moreover, it has been shown that $\mathrm{A} 1$ neurotoxic reactive astrocytes are induced by activated microglia. Expression of A1 astrocytes was found to be extremely high in neurodegenerative diseases including Huntington's, Alzheimer's, and Parkinson's diseases. ${ }^{45}$ A1 astrocytes were identified by co-immunofluorescence staining for C 3 and GFAP. ${ }^{45}$ In our work, we have demonstrated that administration of BMSCs-Exos suppressed activation of microglia. Therefore, we tested expression of A1 neurotoxic reactive astrocytes to find out whether BMSCsExos could suppress activation of A1 neurotoxic reactive astrocytes, thus suppress neuronal apoptosis and, as a result, promote axonal regeneration and functional recovery after SCI. As shown in Figure 10, the number of C3-positive astrocytes cells was significantly lower in the BMSC-Exos treatment group compared to the SCI group at day 1 post-injury, which validated our hypothesis.

\section{Discussion}

SCI can lead to devastating disabilities, such as severe motor deficits, irreversible blindness, and/or paralysis. ${ }^{2,46}$ It also causes upregulation of extrinsic factors, including CSPGs and MAIs, and activates microglia, which leads to continuous neuroinflammation and the destruction of microvascular vessels at the injured site of the CNS. All these factors may affect functional recovery by limiting axonal outgrowth and preventing reformation of functional synapses. ${ }^{37,47,48}$ Here, we demonstrated that administration of exosomes from BMSCs promotes axonal regeneration by downregulating CSPGs, promoting angiogenesis, and suppressing activation of microglia, thus leading to functional behavioral recovery in rats. Meanwhile, neuronal cell apoptosis was attenuated when administrating BMSC-Exos, which could also contribute to better functional behavioral recovery after SCI. Notably, our work demonstrated that BMSCs-Exos act by suppressing A1 neurotoxic reactive astrocytes activation to reduce neuronal cells apoptosis. As a result, axonal regeneration and functional recovery in SCI were improved.

With the rapid development of cell transplantation treatments, BMSCs have been pursued and attracted attention because of their high self-renewal ability, differentiation ability, and low immunogenicity. ${ }^{14,15}$ It has been demonstrated that BMSCs have unique neuroprotective properties and eventually promote functional recovery after acute SCI. ${ }^{10,11}$ However, it was also put forward that only $1 \%$ of transplanted BMSCs could successfully transfer to their desired target tissue. A large number of transplanted BMSCs got trapped inside the lung or liver after intravenous administration. ${ }^{49}$ In addition, BMSC proliferation and differentiation significantly decreased in age-related disorders. ${ }^{50}$ Thus, there several challenges and issues need to be addressed before clinical application.

Exosomes produced by stem cells have shown great therapeutic effects in some diseases, including osteonecrosis, ischemia, and chronic cutaneous wound. ${ }^{26,27,51-53}$ Besides the promising therapeutic effects, administration of exosomes has the unique advantage that it could avoid all limitations caused by direct stem cells transplantation. Stem cells transplantation therapy may act by a paracrine mechanism. ${ }^{19,20}$ We therefore hypothesized whether direct administration of exosomes derived from BMSCs may overcome the limitations and challenges associated with direct stem cell administration therapy and promote functional outcome after SCI. Here, we performed a series of experiments in vivo and in vitro in order to prove our hypothesis. First, exosomes derived from BMSCs were successfully isolated and identified with a range from 20 to $150 \mathrm{~nm}$ 
in diameter using DLS and were further confirmed by TEM and the specific exosome surface markers, including CD9, CD63, and CD81.

Destruction in microvascular and blood vessels in the injured spinal cord is a severe outcome after SCI given that blood supply is crucial for the spinal cord. ${ }^{39}$ Therefore, it is necessary to reconstruct microvascular and blood vessels in the injured spinal cord in order to achieve axonal regeneration and improved behavioral recovery after SCI. Because angiogenesis involves proliferation, migration, and angiogenic tubule formation of endothelial cells, we evaluated the effects of BMSCs-Exos on functional assessments in vitro. The results in vitro indicated that administration of BMSCs-Exos significantly enhanced the angiogenic activities of HUVECs. Using CD31 immunostaining, we demonstrated that exosome treatment remarkably increased the total amount of blood vessels in the SCI lesion at day 28 post-injury. In addition, expression level of VEGF was enhanced. The combined in vitro and in vivo results indicate that administration of BMSCs-Exos exhibited promising proangiogenic properties.

CNS diseases often involve neuronal cell death, including necrosis and apoptosis. ${ }^{54}$ To restore functional and pathological recovery after SCI, neuronal apoptosis is regulated by caspase, Bax, and Bcl-2. ${ }^{55}$ After neuronal injury, the proapoptotic proteins, Bax, cleaved-caspase-3, and caspase-9 were shown to be upregulated, whereas the anti-apoptotic protein, Bcl-2, was downregulated. ${ }^{56,57}$ Our TUNEL assay and flow cytometry results in vitro demonstrated that co-incubation with BMSCs-Exos led to less apoptosis of neuronal cells in the GLU-induced excitotoxicity model, which was also confirmed by our western blot results. Both results in vitro indicated that administration of BMSCs-Exos could protect neuronal cells from GLU-induced apoptosis and excitotoxicity. To further confirm this antiapoptotic mechanism, we evaluated the extent of cell apoptosis in the peritraumatic area of the spinal cord by TUNEL in vivo. As expected, the in vivo TUNEL assay confirmed that BMSCs-Exos could effectively prevent cell apoptosis in the injured spinal cord, which further confirmed our in vitro results. Moreover, western blot results showed that expression level of proapoptotic-related markers in the injured spinal cord was downregulated, whereas the antiapoptotic marker was upregulated.

Traumatic SCI was reported to induce a complex neuroinflammation reaction, including activation of microglia and upregulation of pro-neuroinflammatory cytokines such as TNF- $\alpha$, IL- $1 \beta$, and IL-6. ${ }^{37,58}$ When we added LPS to active microglia in vitro, inflammation was induced. By examining the concentration of $\mathrm{NO}$ production day by day, we found that the production of NO by microglia was downregulated upon incubation with BMSCs-Exos compared to the LPS group. In addition, in our present in vivo study, we tested expression level of proinflammatory cytokines and found that the inflammation was markedly suppressed after BMSCs-Exos administration. By using immunostaining with CD68, representing active microglia, we observed a significant decrease in $\mathrm{CD} 68^{+}$ numbers when treated with BMSCs-Exos at both day 1 and day 28 after SCI compared to the SCI group, suggesting that BMSCs-Exos administration reduced neuroinflammation. All of these results in vitro and in vivo demonstrated that BMSCs-Exos treatment played an anti-inflammatory role in SCI.

Recent studies have identified at least two types of reactive astrocytes: CNS injury-preventing A1 astrocytes and injurypromoting A2 astrocytes. ${ }^{42-45}$ Liddelow and colleagues ${ }^{45}$ proposed that active microglia may convert reactive astrocyte into a neurotoxic state, additionally accompanied by inherent inflammatory cells present in the neural tissue. To confirm their hypothesis, they activated microglial release of molecular cytokines, including
IL1 $\beta$, TNF- $\alpha$, and C1q, which are required and sufficient to drive astrocytes into an A1 neurotoxic and reactive state by LPS treatment. ${ }^{45}$ Using CD68 immunostaining (representing active microglia), we found that administration of BMSCs-Exos effectively suppressed activation of microglia. We therefore wondered whether administration of BMSCs-Exos was able to suppress A1 neurotoxic reactive astrocytes activation and result in decreased neuronal cells apoptosis. By co-immunostaining of GFAP and C3, we showed that the number of A1 astrocytes in the exosome treatment group was remarkably decreased compared to the SCI group at day 1 . To our knowledge, this is the first study elucidating the potential role of BMSCs-Exos in the treatment of SCI and the potential underlying neuroinflammatory mechanisms. However, because of the complexity of astrocytes, we still know little about their normal neuronal development, synapse formation, and proper propagation of action potential change in response to acute SCI. ${ }^{59,60}$ In addition, although BMSCs-Exos show promise in treating SCI, future studies could benefit from tracing and assessing the actual BMSCs-Exos that reach the target SCI lesion.

In conclusion, this study was the first to demonstrate that BMSCs-Exos could effectively promote blood vessel formation, suppress glial scars, reduce neuronal cells apoptosis, decrease inflammatory response, and promote axonal regeneration and, as a result, promote functional behavioral recovery after acute SCI. In particular, one of the underlying mechanisms may be the suppression of the activation of A1 neurotoxic reactive astrocytes. Taken together, these results suggest that the application of exosomes derived from BMSCs may be a promising strategy for SCI.

\section{Acknowledgments}

This work was sponsored by the National Natural Science Foundation of China (grant no.: 81772352), Wu Jieping Foundation (grant no.: 320-2745-16-117), the "333 High Level Talents Project" in Jiangsu Province, China (grant no.; BRA2016512), and the Jiangsu Province Six Talents Peak from Department of Human Resources, Social Security Office of Jiangsu Province, China (grant no.: 2014-WSN-012).

\section{Author Disclosure Statement}

No competing financial interests exist.

\section{References}

1. Fitzharris, M., Cripps, R.A., and Lee, B.B. (2014). Estimating the global incidence of traumatic spinal cord injury. Spinal Cord 52, 117122.

2. Giger, R.J., Hollis, E.R. II, and Tuszynski, M.H. (2010). Guidance molecules in axon regeneration. Cold Spring Harb. Perspect. Biol. 2, a001867.

3. Schwab, M.E., and Bartholdi, D. (1996). Degeneration and regeneration of axons in the lesioned spinal cord. Physiol. Rev. 76, 319-370.

4. Burda, J.E., and Sofroniew, M.V. (2014). Reactive gliosis and the multicellular response to CNS damage and disease. Neuron 81, 229248.

5. Bradbury, E.J., Moon, L.D., Popat, R.J., King, V.R., Bennett, G.S., Patel, P.N., Fawcett, J.W., and McMahon, S.B. (2002). Chondroitinase $\mathrm{ABC}$ promotes functional recovery after spinal cord injury. Nature 416, 636-640.

6. Mothe, A.J., and Tator, C.H. (2012). Advances in stem cell therapy for spinal cord injury. J. Clin. Invest. 122, 3824-3834.

7. Shumsky, J.S., Tobias, C.A., Tumolo, M., Long, W.D., Giszter, S.F. and Murray, M. (2003). Delayed transplantation of fibroblasts genetically modified to secrete BDNF and NT-3 into a spinal cord injury site is associated with limited recovery of function. Exp. Neurol. 184, 114-130. 
8. Liu, D., Jiang, T., Cai, W., Chen, J., Zhang, H., Hietala, S., Santos, H.A., Yin, G., and Fan, J. (2016). An in situ gelling drug delivery system for improved recovery after spinal cord injury. Adv. Healthc. Mater. 5, 1513-1521.

9. Liu, D., Chen, J., Jiang, T., Li, W., Huang, Y., Lu, X., Liu, Z., Zhang, W., Zhou, Z., Ding, Q., Santos, H.A., Yin, G., and Fan, J. (2018). Biodegradable spheres protect traumatically injured spinal cord by alleviating the glutamate-induced excitotoxicity. Adv. Mater. 30, e1706032.

10. Varma, A.K., Das, A., Wallace, G.T., Barry, J., Vertegel, A.A., Ray, S.K., and Banik, N.L. (2013). Spinal cord injury: a review of current therapy, future treatments, and basic science frontiers. Neurochem. Res. 38, 895-905.

11. Chopp, M., Zhang, X.H., Li, Y., Wang, L., Chen, J., Lu, D., Lu, M., and Rosenblum, M. (2000). Spinal cord injury in rat: treatment with bone marrow stromal cell transplantation. Neuroreport 11, 3001-3005.

12. Feron, F., Perry, C., Cochrane, J., Licina, P., Nowitzke, A., Urquhart, S., Geraghty, T., and Mackay-Sim, A. (2005). Autologous olfactory ensheathing cell transplantation in human spinal cord injury. Brain 128, 2951-2960.

13. Ide, C., Nakai, Y., Nakano, N., Seo, T.B., Yamada, Y., Endo, K., Noda, T., Saito, F., Suzuki, Y., Fukushima, M., and Nakatani, T. (2010). Bone marrow stromal cell transplantation for treatment of subacute spinal cord injury in the rat. Brain Res. 1332, 32-47.

14. Cizkova, D., Novotna, I., Slovinska, L., Vanicky, I., Jergova, S., Rosocha, J. and Radonak, J. (2011). Repetitive intrathecal catheter delivery of bone marrow mesenchymal stromal cells improves functional recovery in a rat model of contusive spinal cord injury. J. Neurotrauma 28, 1951-1961.

15. Nakajima, H., Uchida, K., Guerrero, A.R., Watanabe, S., Sugita, D., Takeura, N., Yoshida, A., Long, G., Wright, K.T., Johnson, W.E., and Baba, H. (2012). Transplantation of mesenchymal stem cells promotes an alternative pathway of macrophage activation and functional recovery after spinal cord injury. J. Neurotrauma 29, 1614-1625.

16. Balsam, L.B., Wagers, A.J., Christensen, J.L., Kofidis, T., Weissman, I.L., and Robbins, R.C. (2004). Haematopoietic stem cells adopt mature haematopoietic fates in ischaemic myocardium. Nature 428, $668-673$.

17. Jeong, J.O., Han, J.W., Kim, J.M., Cho, H.J., Park, C., Lee, N., Kim, D.W., and Yoon, Y.S. (2011). Malignant tumor formation after transplantation of short-term cultured bone marrow mesenchymal stem cells in experimental myocardial infarction and diabetic neuropathy. Circ. Res. 108, 1340-1347.

18. Rubio, D., Garcia-Castro, J., Martin, M.C., de la Fuente, R., Cigudosa, J.C., Lloyd, A.C., and Bernad, A. (2005). Spontaneous human adult stem cell transformation. Cancer research 65, 3035-3039.

19. Katsuda, T., Kosaka, N., Takeshita, F. and Ochiya, T. (2013). The therapeutic potential of mesenchymal stem cell-derived extracellular vesicles. Proteomics 13, 1637-1653.

20. Ratajczak, M.Z., Jadczyk, T., Pedziwiatr, D., and Wojakowski, W. (2014). New advances in stem cell research: practical implications for regenerative medicine. Pol. Arch. Med. Wewn. 124, 417-426.

21. Properzi, F., Ferroni, E., Poleggi, A., and Vinci, R. (2015). The regulation of exosome function in the CNS: implications for neurodegeneration. Swiss Med. Wkly. 145, w14204.

22. Azmi, A.S., Bao, B., and Sarkar, F.H. (2013). Exosomes in cancer development, metastasis, and drug resistance: a comprehensive review. Cancer Metastasis Rev. 32, 623-642.

23. Breakefield, X.O., Frederickson, R.M., and Simpson, R.J. (2011). Gesicles: microvesicle "cookies" for transient information transfer between cells. Mol. Ther. 19, 1574-1576.

24. Chaput, N., and Thery, C. (2011). Exosomes: immune properties and potential clinical implementations. Semin. Immunopathol. 33, 419-440.

25. Huang, J.H., Yin, X.M., Xu, Y., Xu, C.C., Lin, X., Ye, F.B., Cao, Y., and Lin, F.Y. (2017). Systemic administration of exosomes released from mesenchymal stromal cells attenuates apoptosis, inflammation, and promotes angiogenesis after spinal cord injury in rats. J. neurotrauma 34, 3388-3396.

26. Tao, S.C., Yuan, T., Zhang, Y.L., Yin, W.J., Guo, S.C., and Zhang, C.Q. (2017). Exosomes derived from miR-140-5p-overexpressing human synovial mesenchymal stem cells enhance cartilage tissue regeneration and prevent osteoarthritis of the knee in a rat model. Theranostics 7, 180-195.

27. Guo, S.C., Tao, S.C., Yin, W.J., Qi, X., Yuan, T., and Zhang, C.Q. (2017). Exosomes derived from platelet-rich plasma promote the re- epithelization of chronic cutaneous wounds via activation of YAP in a diabetic rat model. Theranostics 7, 81-96.

28. Xin, H., Li, Y., Cui, Y., Yang, J.J., Zhang, Z.G., and Chopp, M. (2013). Systemic administration of exosomes released from mesenchymal stromal cells promote functional recovery and neurovascular plasticity after stroke in rats. J. Cereb. Blood Flow Metab. 33, 17111715.

29. Kim, D.K., Nishida, H., An, S.Y., Shetty, A.K., Bartosh, T.J., and Prockop, D.J. (2016). Chromatographically isolated $\mathrm{CD}^{+} 3^{+} \mathrm{CD} 81^{+}$ extracellular vesicles from mesenchymal stromal cells rescue cognitive impairments after TBI. Proc. Natl. Acad. Sci. U. S. A. 113, 170175 .

30. Villarroya-Beltri, C., Gutierrez-Vazquez, C., Sanchez-Madrid, F., and Mittelbrunn, M. (2013). Analysis of microRNA and protein transfer by exosomes during an immune synapse. Methods Mol. Biol. 1024, 4151.

31. Montecalvo, A., Larregina, A.T., and Morelli, A.E. (2013). Methods of analysis of dendritic cell-derived exosome-shuttle microRNA and its horizontal propagation between dendritic cells. Methods Mol. Biol. 1024, 19-40.

32. Takei, N., Numakawa, T., Kozaki, S., Sakai, N., Endo, Y., Takahashi, M., and Hatanaka, H. (1998). Brain-derived neurotrophic factor induces rapid and transient release of glutamate through the non-exocytotic pathway from cortical neurons. J. Biol. Chem. 273, 27620-27624.

33. Tropel, P., Noel, D., Platet, N., Legrand, P., Benabid, A.L., and Berger, F. (2004). Isolation and characterisation of mesenchymal stem cells from adult mouse bone marrow. Exp. Cell Res. 295, 395-406.

34. Gingras, M., Gagnon, V., Minotti, S., Durham, H.D., and Berthod, F. (2007). Optimized protocols for isolation of primary motor neurons, astrocytes and microglia from embryonic mouse spinal cord. J. Neurosci. Methods 163, 111-118.

35. Zhang, J., Guan, J., Niu, X., Hu, G., Guo, S., Li, Q., Xie, Z., Zhang, C., and Wang, Y. (2015). Exosomes released from human induced pluripotent stem cells-derived MSCs facilitate cutaneous wound healing by promoting collagen synthesis and angiogenesis. J. Transl. Med. 13, 49.

36. Basso, D.M., Beattie, M.S., and Bresnahan, J.C. (1995). A sensitive and reliable locomotor rating scale for open field testing in rats. J. Neurotrauma 12, 1-21.

37. Hausmann, O.N. (2003). Post-traumatic inflammation following spinal cord injury. Spinal Cord 41, 369-378.

38. Liu, X.Z., Xu, X.M., Hu, R., Du, C., Zhang, S.X., McDonald, J.W., Dong, H.X., Wu, Y.J., Fan, G.S., Jacquin, M.F., Hsu, C.Y., and Choi, D.W. (1997). Neuronal and glial apoptosis after traumatic spinal cord injury. J. Neurosci. 17, 5395-5406.

39. Figley, S.A., Khosravi, R., Legasto, J.M., Tseng, Y.F., and Fehlings, M.G. (2014). Characterization of vascular disruption and blood-spinal cord barrier permeability following traumatic spinal cord injury. J. Neurotrauma 31, 541-552.

40. Petzold, A. (2005). Neurofilament phosphoforms: surrogate markers for axonal injury, degeneration and loss. J. Neurol. Sci. 233, 183-198.

41. Posmantur, R., Hayes, R.L., Dixon, C.E., and Taft, W.C. (1994). Neurofilament 68 and neurofilament 200 protein levels decrease after traumatic brain injury. J. Neurotrauma 11, 533-545.

42. Zamanian, J.L., Xu, L., Foo, L.C., Nouri, N., Zhou, L., Giffard, R.G., and Barres, B.A. (2012). Genomic analysis of reactive astrogliosis. J. Neurosci. 32, 6391-6410.

43. Yates, D. (2017). Glia: a toxic reaction. Nat. Rev. Neurosci. 18, 130.

44. Burda, J.E., and Sofroniew, M.V. (2017). Seducing astrocytes to the dark side. Cell Res. 27, 726-727.

45. Liddelow, S.A., Guttenplan, K.A., Clarke, L.E., Bennett, F.C., Bohlen, C.J., Schirmer, L., Bennett, M.L., Munch, A.E., Chung, W.S., Peterson, T.C., Wilton, D.K., Frouin, A., Napier, B.A., Panicker, N., Kumar, M., Buckwalter, M.S., Rowitch, D.H., Dawson, V.L., Dawson, T.M., Stevens, B., and Barres, B.A. (2017). Neurotoxic reactive astrocytes are induced by activated microglia. Nature 541, 481-487.

46. Maier, I.C., and Schwab, M.E. (2006). Sprouting, regeneration and circuit formation in the injured spinal cord: factors and activity. Philos. Trans. R Soc. Lond. B Biol. Sci. 361, 1611-1634.

47. Sharma, K., Selzer, M.E., and Li, S. (2012). Scar-mediated inhibition and CSPG receptors in the CNS. Exp. Neurol. 237, 370-378.

48. Yiu, G., and He, Z. (2006). Glial inhibition of CNS axon regeneration. Nat. Rev. Neurosci. 7, 617-627.

49. Phinney, D.G., and Prockop, D.J. (2007). Concise review: mesenchymal stem/multipotent stromal cells: the state of transdifferentia- 
tion and modes of tissue repair-current views. Stem Cells 25, 28962902.

50. Xin, Y., Wang, Y.M., Zhang, H., Li, J., Wang, W., Wei, Y.J. and Hu, S.S. (2010). Aging adversely impacts biological properties of human bone marrow-derived mesenchymal stem cells: implications for tissue engineering heart valve construction. Artif. Organs 34, 215-222.

51. Qi, X., Zhang, J., Yuan, H., Xu, Z., Li, Q., Niu, X., Hu, B., Wang, Y., and Li, X. (2016). Exosomes secreted by human-induced pluripotent stem cell-derived mesenchymal stem cells repair critical-sized bone defects through enhanced angiogenesis and osteogenesis in osteoporotic rats. Int. J. Biol. Sci. 12, 836-849.

52. Liu, X., Li, Q., Niu, X., Hu, B., Chen, S., Song, W., Ding, J., Zhang, C., and Wang, Y. (2017). Exosomes secreted from human-induced pluripotent stem cell-derived mesenchymal stem cells prevent osteonecrosis of the femoral head by promoting angiogenesis. Int. J. Biol. Sci. 13, 232-244.

53. Hu, G.W., Li, Q., Niu, X., Hu, B., Liu, J., Zhou, S.M., Guo, S.C., Lang, H.L., Zhang, C.Q., Wang, Y., and Deng, Z.F. (2015). Exosomes secreted by human-induced pluripotent stem cell-derived mesenchymal stem cells attenuate limb ischemia by promoting angiogenesis in mice. Stem Cell Res. Ther. 6, 10.

54. Yakovlev, A.G., and Faden, A.I. (2004). Mechanisms of neural cell death: implications for development of neuroprotective treatment strategies. NeuroRx 1, 5-16.

55. Liu, S., Sarkar, C., Dinizo, M., Faden, A.I., Koh, E.Y., Lipinski, M.M. and Wu, J. (2015). Disrupted autophagy after spinal cord injury is associated with ER stress and neuronal cell death. Cell Death Dis. 6, e1582.

56. Seki, T., Hida, K., Tada, M., Koyanagi, I., and Iwasaki, Y. (2003) Role of the bcl-2 gene after contusive spinal cord injury in mice. Neurosurgery 53, 192-198; discussion, 198.

57. Springer, J.E., Azbill, R.D., and Knapp, P.E. (1999). Activation of the caspase-3 apoptotic cascade in traumatic spinal cord injury. Nat. Med. 5, 943-946.

58. Donnelly, D.J., and Popovich, P.G. (2008). Inflammation and its role in neuroprotection, axonal regeneration and functional recovery after spinal cord injury. Exp. Neurol. 209, 378-388.

59. Pekny, M., Pekna, M., Messing, A., Steinhauser, C., Lee, J.M., Parpura, V., Hol, E.M., Sofroniew, M.V., and Verkhratsky, A. (2016). Astrocytes: a central element in neurological diseases. Acta Neuropathol. 131, 323-345.
60. Ben Haim, L., and Rowitch, D.H. (2017). Functional diversity of astrocytes in neural circuit regulation. Nat. Rev. Neurosci. 18, 31-41.

Address correspondence to:

Weihua Cai, PhD

Department of Orthopaedics

The First Affiliated Hospital of Nanjing Medical University

300 Guangzhou Road

Nanjing 210029

Jiangsu Province

China

E-mail: caiwhspine@sina.com

or

Jin Fan, PhD

Department of Orthopaedics

The First Affiliated Hospital of Nanjing Medical University

300 Guangzhou Road

Nanjing 210029

Jiangsu Province

China

E-mail: fanjin@njmu.edu.cn

or

Jian Chen, PhD

Department of Orthopaedics

The First Affiliated Hospital of Nanjing Medical University

300 Guangzhou Road

Nanjing 210029

Jiangsu Province

China

E-mail: cbccj@sina.com 